\title{
Spatial vs. Non-Spatial Transboundary Pollution Control in a Class of Cooperative and Non-Cooperative Dynamic Games ${ }^{\text {th }}$
}

\author{
Javier de Frutos ${ }^{\mathrm{a}, 1}$, Guiomar Martín-Herrán ${ }^{\mathrm{a}, 2, *}$ \\ ${ }^{a}$ IMUVA, Universidad de Valladolid. Spain.
}

\begin{abstract}
We analyze a transboundary pollution differential game where, in addition to the standard temporal dimension, a spatial dimension is introduced to capture the geographical relationships among regions. Each region behaves strategically and maximizes its welfare net of environmental damage caused by the pollutant stock. The emission-output ratio is reduced by investment in region specific clean technology which evolves over time. The spatio-temporal dynamics of the pollutant stock is described by a parabolic partial differential equation. Using aggregate variables we study the feedback Nash equilibrium of a discrete-space model which could be seen as a space discretization of the continuous-space model. The discrete-space model presents the three main features of the original formulation: the model is truly dynamic; the agents behave strategically; and the model incorporates spatial aspects. For special functional forms previously used in the literature we analytically characterize the feedback Nash equilibrium and evaluate the impact of the introduction of the spatial dimension in the economic-environmental model. We show that our spatial model is a generalization of the model that disregards the spatial aspects. We analytically show that as the parameter describing how pollution diffuses among regions tends to infinity the equilibrium policies converge to those in the non-spatial setting. In the non-cooperative framework the spatially non-myopic behavior prescribes lower equilibrium emission rates, and consequently a lower global pollution stock. This is compatible with greater long-run welfares. In the cooperative framework, although the strategic interaction among the players does not exist, the only decision-maker still makes spatially strategic decisions.
\end{abstract}

Keywords: OR in Environment and Climate Change, Transboundary Pollution, Spatial Dynamics, Spatially Distributed Controls, Differential Games, Parabolic Differential Equations.

\footnotetext{
The authors are grateful to three anonymous reviewers for valuable comments and suggestions on an earlier draft of this paper. The authors would like to thank participants at EAERE 2017 (Athens), 11th International Society on Dynamic Games Workshop (Warsaw), SING13 (Paris), Second AERNA Workshop on Game Theory and Environment (Madrid).

* Corresponding author. Departamento de Economía Aplicada (Matemáticas), Universidad de Valladolid, Avda. Valle de Esgueva, 6, 47011, Valladolid, Spain. Tel.: +34 983423330 Fax: +34 983 423299;

Email addresses: frutos@mac.uva.es (Javier de Frutos), guiomar@eco.uva.es (Guiomar Martín-Herrán)

${ }^{1}$ This research is partially supported by Spanish MINECO/AEI under project MTM2016-78995-P and by Junta de Castilla y León VA105G18 and VA024P17 co-financed by FEDER funds (EU).

${ }^{2}$ This research is partially supported by Spanish MINECO under projects ECO2014-52343-P and ECO2017-82227P (AEI) and by Junta de Castilla y León VA105G18 and VA024P17 co-financed by FEDER funds (EU).
} 


\section{Introduction}

A review of the literature on dynamic models proposed for studying economic and environmental problems clearly shows that these models focus on the temporal aspect and ignore the spatial aspect. The addition of the spatial aspect obviously enriches the model and its possible predictions, but in turn leads to greater technical difficulties in its analysis. However, recently some authors have added the spatial dimension in the analysis of different economic problems such as allocation of economic activity or technological diffusion (Brito (2004), Boucekkine et al. (2009, 2013a, 2013b), Camacho et al. (2008), Brock \& Xepapadeas (2008a), Desmet \& Rossi-Hansberg (2010), Brock et al. (2014a) and Fabbri (2016)) or environmental and climate problems (Brock \& Xepapadeas (2008b, 2010), Brock et al. (2014b), Camacho \& Pérez-Barahona (2015), Xepapadeas (2010), Anita et al. (2013), Desmet \& Rossi-Hansberg (2015), La Torre et al. (2015) and De Frutos \& Martín-Herrán (2017)). All these papers (except De Frutos \& Martín-Herrán (2017)) analyze finite or infinite time optimal control problems extended to infinite dimensional state space and focus on the problem of a social planner. The social planner allocates resources to maximize the present value of an objective over the entire spatial domain taking into account the spatio-temporal evolution of the state variable. To the best of our knowledge there is only one recent study (De Frutos \& Martín-Herrán (2017)) that considers agents who behave both dynamically and strategically and that takes into account both the spatial and temporal dimension of the problem. The present paper tries to contribute to this very limited literature and analyzes an intertemporal transboundary pollution dynamic game where the pollution stock diffuses over a continuum of spatial sites and there are strategic interactions among the decision-makers.

Other contributions which explore the spatial dimension in environmental economics can be found in Anita et al. (2013, 2015), Brock et al. (2014b), Camacho \& Pérez-Barahona (2015), Desmet \& Rossi-Hansberg (2015), La Torre et al. (2015) and Augeraud-Véron et al. (2017).

Brock et al. (2014b) review the applications of optimal control of diffusive transport processes to environmental and climate problems in economics. Anita et al. (2013) analyze the large-time behavior of a spatially structured economic growth model coupling physical capital accumulation and pollution diffusion. Anita et al. (2015) ad to the previous model a possible taxation based on the amount of produced pollution. The taxation rate depends upon the level of pollution at each spatial location and time. La Torre et al. (2015) extend the analysis in Anita et al. (2013) by introducing abatement activities. They introduce a spatial component in the Solow model and in the Ramsey model and analyze the spatio-temporal dynamics through numerical simulations. Desmet \& Rossi-Hansberg (2015) analyze the geographic impact of climate change through a model featuring two externalities: technology diffusion and emission from energy used in production. Camacho \& Pérez-Barahona (2015) analyze optimal land use from a social planner's point of view who decides the land use activities taking into account that local actions affect the whole space because pollution flows across locations resulting on both local and global damages.

All these contributions focus on the problem of a social planner who allocates resources and hence, disregard the strategic interactions among different decision-makers. These strategic interactions are taken into account in the dynamic game with spatial effects analyzed in De Frutos \& Martín-Herrán (2017). This last paper studies dynamic optimization for the pollution control in a spatial setting with strategic agents and focuses on the equilibrium emission strategies in a multi- 
regional setting. Each economic agent responsible for controlling the emissions at each region takes into account the spatial transport phenomena across space when making the emission decisions at this region in order to maximize his profits.

The present paper shares the main general objective with De Frutos \& Martín-Herrán (2017): to investigate the impact of the strategic and spatial dynamic behaviour of the economic agents responsible for controlling the emissions of pollutant on the design of equilibrium environmental policies. However, the functional specifications in the present work allow us to analytically treat the conditions that characterize the Markov-perfect Nash equilibria of the space-discretized differential game. Conversely, in De Frutos \& Martín-Herrán (2017) the space-discretized differential game is solved using a numerical algorithm adapted from De Frutos \& Martín-Herrán (2015) and the results are illustrated by means of numerical experiments even for the simplest case of two regions. Furthermore, our present functional specification allows us, first, to introduce the possibility of investment in clean technology in order to reduce the emission-output ratio and hence, to analyze how the availability of new technology could affect the optimal emission strategies and the stock of pollution. Second, the present specification allows us to answer our main research question. We discuss the differences in optimal policies and value functions between models that consider the spatial dimension (finite diffusion parameter) and models that do not consider the spatial dimension. We show that our spatial model is a generalization of the standard dynamic model that does not take into account the spatial dimension, in the sense that the behavior of the environmental variables at the equilibrium in the non-spatial setting can be reproduced as a limit case of the spatial setting; in particular, when the parameter describing how pollution diffuses among regions tends to infinity and the stocks of pollution in both regions are instantaneously mixed, which is the main hypothesis made in the non-spatial differential game.

Our paper contributes, on the one hand, to the literature on spatial economics, and more specifically, to the pollution control in a spatial setting previously described by adding the strategic behavior of economic agents. On the other hand, to the literature on transboundary pollution dynamic games (see, for example, Jørgensen et al. (2010) for a survey of this literature) by adding the spatial aspect.

The main objective of the present paper is to evaluate the effect of the strategic and spatially dynamic behaviour of the agents responsible for controlling the emissions of pollutant on the design of equilibrium strategies. Specifically, we aim at comparing the equilibrium strategies, long-run pollution stocks and long-run discounted net welfare of a transboundary pollution dynamic game when the spatial transport phenomena is either taken into account or is ignored. This analysis is carried out both for a non-cooperative and a cooperative formulation of the dynamic game.

The model is originally stated in continuous space and continuous time with two spatial dimensions and one temporal dimension. There are $J$ players and each player decides the emission level and the investment in clean technology in order to maximize the present value of benefits net of environmental damages due to the concentration of pollutants over his spatial domain. The emission-output ratio in each region rather than assumed to be constant as in most of the papers of the literature of environmental dynamic games (Jørgensen et al. (2010)), is assumed to be a decreasing and strictly convex function of the stock of clean technology of this region. The maximization problem of each region is subject to the temporal evolution of the stock of clean technology 
which is assumed to be region specific (Jørgensen \& Zaccour (2001)) and to the spatio-temporal evolution of the stock of a pollutant. The spatio-temporal evolution of the stock of a pollutant is described by a diffusion partial differential equation (PDE) and general boundary conditions are assumed. This PDE is a generalization of the PDE describing this evolution in one of the examples presented in Brock et al. (2014b). While their specification is one-dimensional, ours is two-dimensional allowing to better describe the geographical or spatial aspect of the problem.

It is worth noting that in order to maintain the model simple and to focus on the spatial dimension of pollution diffusion we do not allow the spatial diffusion of clean technology. This is the standard assumption in the literature when spatial effects are disregarded. Therefore, in order to emphasize the spatial aspect of pollution diffusion, we consider a spatial model where agents behave strategically, the clean technology evolves over time and the pollution stock evolves across space and over time.

Our original specification is a $J$-player differential game. Each player aims at maximizing his profits net of environmental damages by choosing his level of emission and investment in new technology at each spatial point in his region and at each time. When making his decisions, each player takes into account the temporal evolution of his stock of clean technology described by a ordinary differential equation (ODE) and the spatio-temporal evolution of the stock of a pollutant described by a partial differential equation (PDE).

Along the same lines as in De Frutos \& Martín-Herrán (2017) we apply a spatial discretization approach to simplify the model and characterize the equilibrium outcomes of the transboundary pollution dynamic game with spatial effects. In the space-discretized model each player decides the average total emission in his region and his investment in clean technology taking into account the time evolution of the average pollution in each region and the stock of clean technology. The new formulation has $2 J$ state variables described by a system of $2 J$ ordinary differential equations (ODEs). A similar spatial discretization approach has been recently proposed in Graß \& Uecker (2017) in a optimal control framework and applied to the analysis of a shallow lake model with diffusion. The structure of our space-discretized formulation is similar to that proposed in Mäler \& Zeeuw (1998) to analyze an acid rain differential game.

The space-discretized model is exactly solved, unlike the model in De Frutos \& Martín-Herrán (2017) that has to be numerically solved using a numerical algorithm. From our results we can conclude that the space-discretized formulation is a good first approach to characterize the equilibrium outcomes of the transboundary pollution dynamic game with spatial effects. Our analytical results show that the space-discretized model is a clear generalization of the model which ignores the spatial transport phenomena. Specifically, we analytically prove that for a two-player setting and both for non-cooperative and cooperative frameworks the traditional equilibrium policies derived ignoring the spatial dimension can be reproduced as a limit case of the space-discretized formulation. The limit case is described by the diffusion pollution parameter tending to infinity, when the stocks of pollution in both regions are instantaneously mixed, the assumption implicitly considered in the non-spatial dynamic game.

Our analytical results show how the equilibrium emission policies in a spatial context differ from those characterized ignoring the spatial dimension. If the regions behave myopically, disregarding the spatial dimension of the problem, then they are considering that the stocks of pollution are 
instantaneously mixed. The consequence of this hypothesis in the non-cooperative framework is that the players behave as free-riders. Each region emits at a greater level with respect to the case without perfect mixing of the pollution stocks, because it recognizes that the effect of its own emissions is instantaneously shared with the other region. The greater equilibrium emission rates lead to a greater global pollution stock. On the contrary, the spatially non-myopic players, who assume that the pollution stocks are mixed at a finite speed, optimally decide lower equilibrium emission rates, resulting in a lower global pollution stock. Interestingly, this is compatible with greater long-run welfares. Consequently, in the symmetric case the spatially myopic players underestimate their long-run welfares with respect to their welfares when the regions acknowledge the geographical aspect of the problem.

In the cooperative framework, although the strategic interaction among the players does not exist, the only decision-maker still makes spatially strategic decisions. If both regions are completely symmetric, the stocks of pollution are equally valued by the only decision-maker. Therefore, he is only interested in the average and his optimal emission decisions are identical regardless of whether he behaves myopically or non-myopically from a spatial point of view. In consequence, the long-run welfares are the same in both cases. This result is different from the result obtained in the noncooperative scenario. On the contrary, if the only factor of asymmetry is the environmental damage cost, without a perfect mixing of the pollution stocks, the social planner will decide strategically and prescribe greater emissions in the region with a lower environmental cost. However, with perfect mixing of the pollution stocks, the optimal emission levels will be identical among regions regardless of the environmental damage cost parameter.

Overall, the geographical aspects are essential ingredients when determining the equilibrium values of the emission levels under both cooperative and non-cooperative frameworks.

The paper is organized as follows. In the next section we present the multiregional spatially distributed control of pollution formulated initially as a continuous-space model, and in a second step, as a discrete-space model. Section 3 analyzes a particular specification of the model and presents the characterization of the Markov-perfect Nash equilibrium of the model. Section 4 analytically shows for the 2-player case the main differences between the environmental policies in the formulation with and without spatial effects. Section 5 revisits this comparison but for a cooperative setting, where there is a unique decision-maker and the strategic interactions among the players disappear. Section 6 is devoted to present some concluding remarks. The proofs are collected in the Appendix.

\section{The model}

The following table summarizes the notations used along the paper. 


\begin{tabular}{ll}
\hline$\Omega$ & planar domain of interest \\
$J$ & number of players \\
$\Omega_{i}$ & region of $\Omega$ under control of player $i, i=1, \ldots, J$ \\
$P(\boldsymbol{x}, t)$ & pollution stock at $\boldsymbol{x} \in \Omega$ and time $t>0$ \\
$Y_{i}(\boldsymbol{x}, t)$ & production of region $i$ at $\boldsymbol{x} \in \Omega$ and time $t>0$ \\
$E_{i}(\boldsymbol{x}, t)$ & emissions of region $i$ at $\boldsymbol{x} \in \Omega$ and time $t>0$ \\
$K_{i}(t)$ & stock of clean technlogy of region $i$ at time $t>0$ \\
$\alpha_{i}\left(K_{i}\right)$ & emissions-output ratio of region $i$ \\
$I_{i}(t)$ & investment in clean technology of region $i$ at time $t>0$ \\
$b(\boldsymbol{x})$ & $\boldsymbol{x}$-dependent diffusion coefficient \\
$p_{i}(t)$ & average of pollution stock in region $i$ at time $t>0$ \\
$e_{i}(t)$ & average of emissions in region $i$ at time $t>0$ \\
$\boldsymbol{p}=\left[p_{1}, \ldots, p_{J}\right]^{T}$ & vector of agregated stocks of pollution \\
$\boldsymbol{e}=\left[e_{1}, \ldots, e_{J}\right]^{T}$ & vector of averaged emissions \\
$\boldsymbol{K}=\left[K_{1}, \ldots, K_{J}\right]^{T}$ & vector of stocks of clean technology \\
$B=\left[b_{i, j}\right]_{i, j=1}^{J}$ & $J \times J$ matrix of diffusion coefficients \\
$\rho$ & constant time discount rate \\
$\varphi_{i}$ & environmental damage parameter in region $i$ \\
$c_{i}$ & investment cost parameter in region $i$ \\
$\beta_{i}$ & clean technology parameter in region $i$ \\
$q_{i}$ & aggregated normalized boundary data in region $i$ \\
$d_{i}$ & decay rate of pollution stock in region $i$ \\
$\eta_{i}$ & ratio of emission to pollution stock per unit of time in region $i$ \\
$\mu_{i}$ & \\
$D, \Gamma, \Pi$ &
\end{tabular}

Table1: Notations

Let us denote by $\Omega$ a planar domain (open connected set) which is partitioned in $J$ regions (open connected sets) $\Omega_{j}, j=1, \ldots, J$, such that

$$
\bar{\Omega}=\bigcup_{j=1}^{J} \bar{\Omega}_{j}, \quad \Omega_{i} \cap \Omega_{j}=\emptyset, \quad i \neq j,
$$

where $\bar{\Omega}$ is the closure of $\Omega$. The common boundary between regions $\Omega_{i}$ and $\Omega_{j}$ is given by $\partial_{i j}:=\partial \Omega_{i} \cap \partial \Omega_{j}=\bar{\Omega}_{i} \cap \bar{\Omega}_{j}, i \neq j$.

In each region there is one decision-maker. Player $i$ wishes to choose the rate of pollutant emissions in region $\Omega_{i}$ as well as the investment in clean technology to maximize his own payoff. Therefore, the differential game considers $J$ players (regions) and each player has pollution emissions and investments in clean technology as control variables. The $J$-player differential game is played non-cooperatively. 
Each region $i$ produces a single consumption good. We denote by $Y_{i}(\boldsymbol{x}, t)$ the production of the good at time $t \geq 0$ at the particular point $\boldsymbol{x} \in \Omega$. The instantaneous net social benefits of production of region $i$ are given by $B_{i}\left(Y_{i}(\boldsymbol{x}, t)\right)$, with function $B_{i}$ being increasing and strictly concave. The production of $Y_{i}(\boldsymbol{x}, t)$ generates pollution emissions. Hence, the industrial activities of the regions create pollution as an undesirable by-product. Let us denote by $E_{i}(\boldsymbol{x}, t), i=1, \ldots, J$, the emission rate of region $i$ at time $t \geq 0$ and $\boldsymbol{x} \in \Omega$. It is convenient to think of $E_{i}(\boldsymbol{x}, t)$ and $Y_{i}(\boldsymbol{x}, t)$ as densities of emission rate and production which are distributed along the domain $\Omega$. Also it is convenient to assume that although $E_{i}(\boldsymbol{x}, t)$ and $Y_{i}(\boldsymbol{x}, t)$ are defined for all $\boldsymbol{x} \in \Omega, E_{i}(\boldsymbol{x}, t)=0$, $Y_{i}(\boldsymbol{x}, t)=0$ for $\boldsymbol{x} \notin \Omega_{i}$. Most of the papers of the literature of environmental dynamic games (see Jørgensen et al. (2010) for a survey of dynamic games models used to analyze transboundary pollution problems) assumed a constant emission-output ratio. However, following Ploeg \& Zeeuw (1992) and Jørgensen \& Zaccour (2001) we assume that the emission-output ratio is dependent on the stock of clean technology of region $i$ at time $t$, denoted by $K_{i}(t)$. The idea is that the emission-output ratio can be reduced by investment in clean technology. Hence, the emission rate $E_{i}(\boldsymbol{x}, t)$ resulting from production of region $i$ is given by

$$
E_{i}(\boldsymbol{x}, t)=\alpha_{i}\left(K_{i}(t)\right) Y_{i}(\boldsymbol{x}, t) .
$$

Function $\alpha_{i}$ is a decreasing and strictly convex function of the stock of clean technology of region $i$ to account for decreasing returns in the investment activities in clean technology. Ploeg \& Zeeuw (1992) assume that the stock of clean technology is public knowledge, while Jørgensen \& Zaccour (2001) consider the case where the stock of clean technology is region specific. We follow this last hypothesis.

As a first step in the analysis and in order to maintain the model simple and to focus on the spatial dimension of pollution diffusion, we do not allow the spatial diffusion of clean technology. With this hypothesis we are assuming that the technology in each region $\Omega_{i}$ is not transferred to region $\Omega_{j}$, with $j \neq i$. Furthermore, the clean technology $K_{i}$ is independent of the particular point $\boldsymbol{x} \in \Omega_{i}$. That is, clean technology is homogeneous within each region $\Omega_{i}$, although it is different between regions. The clean technology is region specific. Therefore, in order to emphasize the spatial aspect of pollution diffusion, we consider a spatial model where agents behave strategically, the clean technology evolves over time and the pollution stock evolves across space and over time.

The dynamics of the stocks of clean technology over time is described by the following differential equations:

$$
\dot{K}_{i}(t)=f\left(I_{i}(t), K_{i}(t)\right), \quad K_{i}(0)=K_{i 0},
$$

where a dot over a variable denotes its derivative with respect to time, $I_{i}(t)$ is the investment in clean technology in region $i$ and $K_{i 0}$ is the initial stock of clean technology in this region. The cost associated with investment in clean technology is denoted by $C_{i}\left(I_{i}(t)\right)$, where function $C_{i}$ is assumed to be increasing and strictly convex.

The emissions accumulate in a stock of pollution denoted by $P(\boldsymbol{x}, t)$ and defined for all $\boldsymbol{x} \in \Omega$. In what follows we denote by $\nabla u$ the spatial gradient of a scalar function $u: \Omega \rightarrow \mathbb{R}$, and by $\nabla \cdot \boldsymbol{u}=$ $\frac{\partial u_{1}}{\partial x}+\frac{\partial u_{2}}{\partial y}$ the divergence of a vectorial function $\boldsymbol{u}=\left[u_{1}, u_{2}\right]: \Omega \rightarrow \mathbb{R}^{2}$. The following parabolic 
partial differential equation describes the spatio-temporal dynamics of the stock of pollution:

$$
\begin{aligned}
& \frac{\partial P}{\partial t}=\nabla \cdot(b \nabla P)-d P+\sum_{j=1}^{J} F_{j}\left(E_{j}(\boldsymbol{x}, t)\right) \mathbf{1}_{\Omega_{j}}, \boldsymbol{x} \in \Omega, \\
& P(\boldsymbol{x}, 0)=P^{0}(\boldsymbol{x}), \quad \boldsymbol{x} \in \Omega, \\
& a(\boldsymbol{x}) P(\boldsymbol{x}, t)+b(\boldsymbol{x}) \nabla P^{T}(\boldsymbol{x}, t) \boldsymbol{n}=a(\boldsymbol{x}) P_{b}(\boldsymbol{x}, t), \quad \boldsymbol{x} \in \partial \Omega .
\end{aligned}
$$

The velocity at which the stock of pollution is diffused away in a particular location $\boldsymbol{x}$ is measured by $b=b(\boldsymbol{x})$, a local diffusion coefficient which is assumed to be a smooth function satisfying $b_{m} \leq b(\boldsymbol{x}) \leq b_{M}$, for all $\boldsymbol{x} \in \Omega$, where $0<b_{m} \leq b_{M}$ are two given constants. The natural decay of the pollutant is represented by the term $d P$, with $d=d(\boldsymbol{x}, t) \geq 0$. In the source term $\sum_{j=1}^{J} F_{j}\left(E_{j}(\boldsymbol{x}, t)\right) \mathbf{1}_{\Omega_{j}}, F_{j}, j=1, \ldots, J$ is a family of non decreasing smooth functions, with $\mathbf{1}_{\Omega_{j}}$ denoting the characteristic function of set $\Omega_{j}$, that is, the function defined to be identically one on $\Omega_{j}$, and zero elsewhere. With this specification, at a given time $t$, each region $i$ emits at rate $E_{i}(\boldsymbol{x}, t)$ in each point $\boldsymbol{x} \in \Omega_{i}$ contributing to enlarge locally the stock of pollution $P(\boldsymbol{x}, t)$. However, the diffusion process modeled by the state equation (3) transfers part of the stock of pollution to the whole region $\Omega$. In consequence, all the regions $\Omega_{j}, j=1, \ldots, J$, are affected by the emissions $E_{i}(\boldsymbol{x}, t)$ of a particular region $\Omega_{i}$. The diffusive character of the state equation implies that the emissions in region $\Omega_{i}$ instantaneously affect each one of the regions $\Omega_{j}, i \neq j$. How much region $j$ is affected by the emissions of region $i$ depends on the time elapsed from the instant when the emissions take place and the distance between regions $i$ and $j$.

The initial distribution of the stock of pollution along region $\Omega$ is described in the second equation in (3) and the boundary condition is stated in the third equation of (3). The boundary condition states that the flux of pollution throughout $\partial \Omega$ is proportional to the difference $P_{b}(\boldsymbol{x})-$ $P(\boldsymbol{x})$, where $P_{b}(\boldsymbol{x})$ is a given function representing the concentration of pollution in the exterior of $\Omega$ and $\boldsymbol{n}$ denotes the normal vector exterior to $\Omega$. Function $a(\boldsymbol{x})$ is a non-negative smooth function that appears after applying Newton's law of diffusion on the boundary of $\Omega$.

The literature has used extensively diffusion models similar to equation (3) to describe mathematically the contaminant transport in different applications such as atmospheric dispersion of pollutants, transport of pollutants in aquifers, groundwater pollution, or porous soil (Stockie 2011).

Player $i, i=1, \ldots, J$ aims at maximizing his payoff

$$
J_{i}\left(E_{1}, \ldots, E_{J}, I_{i}, P_{0}, K_{i 0}\right)=\int_{0}^{+\infty} \int_{\Omega_{i}} e^{-\rho t} G_{i}\left(E_{i}, I_{i}, P, K_{i}\right) \mathrm{d} \boldsymbol{x} \mathrm{d} t
$$

taking into account the dynamics of the stocks of clean technology and the pollution stock given in equations (2) and (3), respectively. In the expression above $\rho>0$ denotes the time-discount rate. This payoff can be understood as an average over $\Omega_{i}$ of a density of revenue represented by $G_{i}\left(E_{i}, I_{i}, P, K_{i}\right)$. We remark that the emissions in region $j, j \neq i$, enter into the objective functional of player $i$ via the state equation (3).

The standard assumption in dynamic pollution games (see, for example, Jørgensen et al. (2010) for a survey of this literature) establishes that the instantaneous welfare of each region is given by a benefit from consumption $\left(B_{i}\left(Y_{i}(\boldsymbol{x}, t)\right)\right)$ minus the cost of the investment in clean technology 
$\left(C_{i}\left(I_{i}(t)\right)\right)$ and the damage caused by the stock of pollution $D_{i}(P(\boldsymbol{x}, t))$. The smooth function $D_{i}$ is commonly assumed in the literature to be convex in its argument. Therefore, the net benefits from consumption have the form

$$
\left(B_{i}\left(Y_{i}\right)-C_{i}\left(I_{i}\right)-D_{i}(P)\right) \mathbf{1}_{\Omega_{i}}
$$

Taking into account the emission-production trade-off function in (1), the instantaneous benefits of region $i$ in (5) can be rewritten in terms of the emission rates and its own stock of clean technology:

$$
G_{i}\left(E_{i}, I_{i}, P, K_{i}\right)=\left(B_{i}\left(\alpha\left(K_{i}\right)^{-1} E_{i}\right)-C_{i}\left(I_{i}\right)-D_{i}(P)\right) \mathbf{1}_{\Omega_{i}}
$$

We restrict the analysis to stationary Markov-perfect Nash equilibria because the strategies supporting this equilibria do not require precommitment to a course of action over time and have been assumed to be a good description of realistic behaviour (see, for example, Haurie et al. (2012) and Jørgensen et al. (2010)). From now on we assume that the dynamic game defined by (2)-(6) has at least one stationary Markov-perfect Nash equilibrium (Başar \& Olsder (1999)). From (2) and (3) it can be easily deduced that the optimal strategies of each region will be independent of the stock of clean technology of the other regions. Therefore, the emission and investment decisions of an agent at any point in time and space only depend on the state of the pollution stock and his own stock of clean technology at that moment and point in space.

In order to answer our main research questions and focus on the comparison of the equilibrium strategies when the spatial aspects are either taking into account or disregarded, we introduce aggregated variables for the environmental variables and reformulate the model. The discretespace model derived using these aggregate variables can be obtained along the same lines as in De Frutos \& Martín-Herrán (2017). The discrete-space model can also be seen as a space discretization of the continuous-space model. It is worth noting that the discrete-space model is truly dynamic, incorporates spatial aspects and the decision makers behave strategically.

The aggregated stock of pollution and the averaged emissions in each region $i$ are defined by

$$
p_{i}(t)=\frac{1}{m_{i}} \int_{\Omega_{i}} P(\boldsymbol{x}, t) \mathrm{d} \boldsymbol{x}, \quad e_{i}(t)=\frac{1}{m_{i}} \int_{\Omega_{i}} E_{i}(\boldsymbol{x}, t) \mathrm{d} \boldsymbol{x}, \quad i=1, \ldots, J,
$$

where $m_{i}$ represents the area of region $\Omega_{i}$.

Let us define

$$
\widehat{G}_{i}\left(e_{i}, I_{i}, p_{i}, K_{i}\right)=m_{i} G_{i}\left(e_{i}, I_{i}, p_{i}, K_{i}\right),
$$

which is an approximation to $\int_{\Omega_{i}} G_{i}\left(E_{i}, I_{i}, P, K_{i}\right) \mathrm{d} \boldsymbol{x}$.

The objective of Player $i$ in the discrete-space model is to maximize the space averaged payoff

$$
\widehat{J}_{i}\left(e_{1}, \ldots, e_{J}, I_{i}, \boldsymbol{p}^{0}, K_{i 0}\right)=\int_{0}^{\infty} e^{-\rho t} \widehat{G}_{i}\left(e_{i}, I_{i}, p_{i}, K_{i}\right) \mathrm{d} t,
$$

taking into account the dynamics of his own stock of clean technology given by (2) and the dynamics of the aggregated stock of pollution in each region described by the following system of ordinary 
differential equations:

$$
m_{i} \dot{p}_{i}=\sum_{\substack{j=1 \\ j \neq i}}^{J} b_{i j}\left(p_{j}-p_{i}\right)+b_{i 0}\left(p_{i 0}-p_{i}\right)-m_{i} \delta_{i} p_{i}+m_{i} F_{i}\left(e_{i}\right), \quad i=1, \ldots, J
$$

System (9) is supplemented with initial conditions given by

$$
p_{i}(0)=\frac{1}{m_{i}} \int_{\Omega_{i}} P_{0}(\boldsymbol{x}) \mathrm{d} \boldsymbol{x}:=p_{i}^{0}, \quad i=1, \ldots, J,
$$

where $P_{0}(\boldsymbol{x})$ is the initial data in (3). From now on we use the notation $\boldsymbol{p}^{0}=\left[p_{1}^{0}, \ldots, p_{J}^{0}\right]^{T}$.

Coefficients $b_{i j}$ measure how fast the pollution spreads across boundary $\partial_{i j}$ between regions $\Omega_{i}$ and $\Omega_{j}$ in absence of external transport phenomena. Of course, it is understood that $b_{i j}=0$ if regions $\Omega_{i}$ and $\Omega_{j}$ have no common boundary and $b_{i j} \neq 0$ in the opposite case when $\partial_{i j} \neq \emptyset$, that is, regions $\Omega_{i}$ and $\Omega_{j}$ share a common boundary. It is implicitly assumed that $b_{i j}=b_{j i}$ for all $i, j$. Region corresponding to index $i=0$ represents the exterior of $\Omega$. The stock of pollution $p_{i 0}$ can be obtained by aggregation on $\partial \Omega_{i} \cap \partial \Omega$ of the boundary data $P_{b}$ in formula (3), so that it is a known function of time in (9). The first two terms in the right hand side of the differential equation in (9) collect the diffusion effect that tends to equilibrate the pollution between regions: the pollution entering $\Omega_{i}$ is proportional to the difference between the stock of pollution in the adjacent regions, the pollution moves from regions with high levels of concentration to regions with low levels of concentration (Flick's law of diffusion). The third term is pollution degradability or

natural degradation of the pollution stock, where $\delta_{i}=1 / m_{i} \int_{\Omega_{i}} d(\boldsymbol{x}) d \boldsymbol{x}$ represents the averaged value of the natural decay of the pollutant. Finally, the fourth term is the flow of emissions where $F_{i}\left(e_{i}\right)$ represents the contribution to the stock of pollution in $\Omega_{i}, p_{i}$, by the averaged emissions in region $i$.

The discrete-space model described by $(2),(8)$ and (9) is a $J$-player infinite horizon differential game with two decision variables for each player (the averaged emission rates in his region and the investment in his own clean technology) and $2 J$ state variables (the stock of clean technology and the averaged stock of pollution in each region) with time evolution described by the system of ODEs in (2) and (9).

\section{A particular specification of the discrete-space model}

We adopt the simplest version of the economics and environment model that still captures the main ingredients of the more general model described in the previous section. First, the strategic behavior of the players, emissions by one player affects the environment of all; and second, the spatial aspect that allows us to analyze how our results compare to those obtained using standard dynamic game models which disregard the spatial dimension of the problem.

From now on we use the special functional forms proposed in Jørgensen \& Zaccour (2001). The functional forms for instantaneous benefits, costs of investment in clean technology, emission-output 
ratio and the damage environmental cost are assumed as follows:

$$
B_{i}\left(Y_{i}\right)=\log \left(Y_{i}\right), C_{i}\left(I_{i}\right)=\frac{1}{2} c_{i} I_{i}^{2}, \alpha_{i}\left(K_{i}\right)=\sigma_{i} e^{-\beta_{i} K_{i}}, D_{i}(P)=\varphi_{i} P, i=1, \ldots, J,
$$

where $c_{i}, \sigma_{i}, \beta_{i}$ and $\varphi_{i}$ are positive constants. ${ }^{3}$

The clean technology stocks evolve in time according to the standard dynamics:

$$
\dot{K}_{i}(t)=I_{i}(t)-\mu_{i} K_{i}(t), \quad K_{i}(0)=K_{i 0}, \quad i=1, \ldots, J,
$$

where $\mu_{i}$ is the rate of depreciation of technology which as usual is assumed to be constant and $K_{i 0}$ is a given initial stock of clean technology for region $i$.

The source function in (9) is given by:

$$
F_{i}\left(e_{i}\right)=\eta_{i} e_{i}, \quad i=1, \ldots, J
$$

Under these hypotheses the $J$-player differential game played over an infinite-time horizon defined by (2), (8) and (9) particularizes as follows: Each region $i$ chooses its control variables, the average emission rate $e_{i}$ and the investment in clean technology $I_{i}$ in order to maximize

$$
\widetilde{J}_{i}\left(e_{i}, I_{i}, \boldsymbol{p}^{0}, K_{i 0}\right)=\int_{0}^{\infty} e^{-\rho t}\left(\log \left(e_{i}\right)+\beta_{i} K_{i}-\frac{1}{2} c_{i} I_{i}^{2}-\varphi_{i} p_{i}\right) \mathrm{d} t
$$

subject to the dynamics of its own stock of clean technology given by (10) and of the aggregated stock of pollution in each region defined by

$$
\dot{p}_{i}=\sum_{\substack{j=1 \\ j \neq i}}^{J} b_{i j}\left(p_{j}-p_{i}\right)-d_{i}\left(p_{i}-q_{i}\right)+\eta_{i} e_{i}, \quad p_{i}(0)=p_{i}^{0}, \quad i=1, \ldots, J
$$

where $d_{i}=\delta_{i}+b_{i 0}$ and $q_{i}=b_{i 0} p_{i 0} / d_{i} . b_{i 0}$ represents how fast the pollution stock in $\Omega_{i}$ spreads out of $\Omega_{i}$ to the exterior of $\Omega$. Parameter $d_{i}$ incorporates the effect in $\Omega_{i}$ of the natural decay rate of pollution (via $\delta_{i}$ ) as well as the effect of the transfer of the pollution stock to the exterior of $\Omega$ (via $b_{i 0}$ ). From now on we assume that $q_{i}, i=1 \ldots, J$, are independent of time and, for the ease of notation, we will denote by $b_{i j}$ the diffusion coefficient once it has been normalized by $m_{i}$, the total area of region $\Omega_{i}$.

Let us note that a constant term $-\log \left(\sigma_{i}\right)$ should appear in the objective (11). Because this term does not affect the optimal policies it has been omitted for simplicity.

Next we will use vectorial notation. Then, we introduce vectors $\boldsymbol{p}=\left[p_{1}, \ldots, p_{J}\right]^{T}, \boldsymbol{K}=$ $\left[K_{1}, \ldots, K_{J}\right]^{T}, \boldsymbol{e}=\left[e_{1}, \ldots, e_{J}\right]^{T}, \boldsymbol{q}=\left[q_{1}, \ldots, q_{J}\right]^{T}$ and diagonal matrices $\Pi=\operatorname{diag}\left(\eta_{1}, \ldots, \eta_{J}\right)$, $\Gamma=\operatorname{diag}\left(\mu_{1}, \ldots, \mu_{J}\right), \mathrm{D}=\operatorname{diag}\left(d_{1}, \ldots, d_{J}\right)$, and $B$ is the symmetric matrix $B=\left[b_{i j}\right]_{i, j=1}^{J}$, with

\footnotetext{
${ }^{3}$ The model can be easily generalized to allow each player to invest in different clean technologies. We opt to present here the simplest version that allows us to answer our research questions.
} 
$b_{i i}=-\sum_{j \neq i} b_{i j}$, so that the dynamics (10)-(12) can be written in the condensed form

$$
\begin{aligned}
\dot{\boldsymbol{K}}(t) & =\boldsymbol{I}(t)-\Gamma \boldsymbol{K}(t), \quad \boldsymbol{K}(0)=\boldsymbol{K}_{0}, \\
\dot{\boldsymbol{p}}(t) & =\mathrm{B} \boldsymbol{p}(t)-\mathrm{D}(\boldsymbol{p}(t)-\boldsymbol{q})+\Pi e(t), \quad \boldsymbol{p}(0)=\boldsymbol{p}_{\mathbf{0}} .
\end{aligned}
$$

With the particular functional forms considered here, the dynamic game belongs to the class of state separable or linear-state differential games. For this class of games it is well-known that the Hamilton-Jacobi-Bellman equations that characterize the non-cooperative feedback Nash equilibrium are satisfied for value functions linear in the state variables, in our case $K_{i}, p_{i}, p_{j}$ for player $i$ (Dockner et al. (2000)). It is well-known that the feedback Nash equilibria in linear-state differential games can be analytically characterized although they are degenerated in the sense that they are constant over time. However, we select this simplest specification in order to clearly identify the impact of introducing spatial diffusion effects (or geographical effects) in equilibrium strategies.

Proposition 1. The equilibrium emission and investment rates of the differential game defined by (10), (11) and (12) are constant and given by:

$$
I_{i}=\frac{M_{i, i}}{c_{i}}, \quad e_{i}=-\frac{1}{\eta_{i} R_{i, i}}
$$

where

$$
M_{i, i}=\frac{\beta_{i}}{\rho+\mu_{i}} \quad \text { and } \quad R_{i, i}=\frac{1}{\varphi_{i}} \boldsymbol{R}_{i}^{T}(\mathrm{~B}-\rho \mathrm{Id}-\mathrm{D}) \boldsymbol{R}_{i}<0, \quad i=1, \ldots, J,
$$

with $\boldsymbol{R}_{\boldsymbol{i}}=\left[R_{i, 1}, \ldots, R_{i, J}\right]^{T}, i=1, \ldots, J$ the solution of the following system of linear equations

$$
(\mathrm{B}-\rho \mathrm{Id}-\mathrm{D}) \boldsymbol{R}_{i}=\varphi_{i} \boldsymbol{u}_{i}, \quad i=1, \ldots, J
$$

where Id denotes the $J \times J$ identity matrix and $\boldsymbol{u}_{i}$ is the $i$-th vector of the usual base of $\mathbb{R}^{J}$.

The discounted net welfare of player $i$ is given by:

$$
V_{i}(\boldsymbol{p}, \boldsymbol{K})=\boldsymbol{M}_{i}^{T} \boldsymbol{K}+\boldsymbol{R}_{\boldsymbol{i}}^{T} \boldsymbol{p}+X_{i},
$$

with $\boldsymbol{M}_{i}=\left[M_{i, 1}, \ldots, M_{i, J}\right]^{T}, M_{i j}=0, j \neq i, M_{i i}$ defined in (16) and

$$
X_{i}=\frac{1}{\rho}\left(\log \left(e_{i}\right)-\frac{1}{2} c_{i} I_{i}^{2}+M_{i, i} I_{i}+\boldsymbol{R}_{i}^{T}(\Pi \boldsymbol{e}+D \boldsymbol{q})\right),
$$

where $e_{i}$ and $I_{i}, i=1, \ldots, J$ are the values given in (15).

The stationary equilibrium of the dynamics is globally asymptotically stable.

\subsection{Numerical examples}

We present now a couple of numerical examples that help us understand the spatial problem of transboundary pollution. These two examples have been chosen in order to illustrate the differences with respect to the equilibrium environmental policies prescribed by the standard dynamic game models which disregard the spatial aspect. 
The parameter values used in the numerical examples are the following:

$$
\eta_{i}=1, \quad \varphi_{i}=1, \quad d=0.5, \quad \rho=0.01, i=1, \ldots, J .
$$

The entries $b_{i j}$ of matrix $B$ are chosen as $b_{i j}=1$ if regions $\Omega_{i}$ and $\Omega_{j}$ are neighbors $\left(\partial_{i j} \neq \emptyset\right)$. Note that with this choice of the parameter values we are highlighting the fact that the players are completely symmetrical in every respect, except in their geographical positions described by the entries $b_{i j}$ of matrix $B$. Recall that $b_{i j}$ indicates how fast pollution spreads across the common boundary between $\Omega_{i}$ and $\Omega_{j}$.

It is worth noting that for this completely symmetric framework the equilibrium emission rates prescribed by the non-spatial dynamic game would be identical for all the regions.
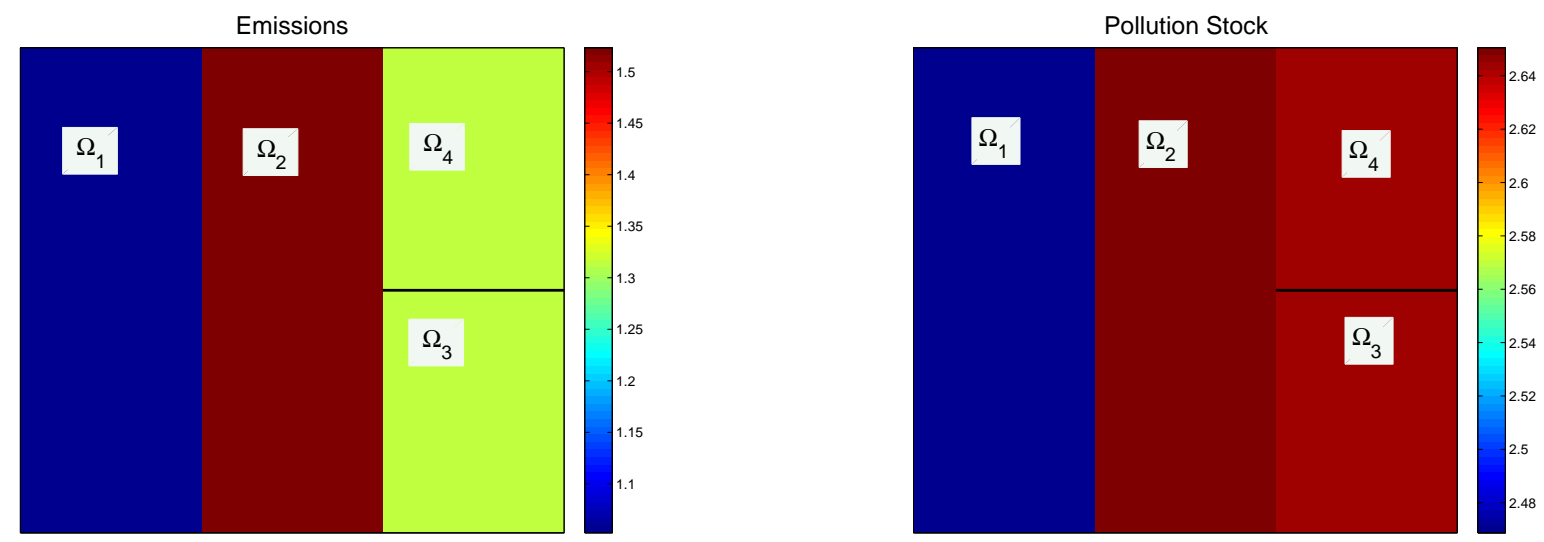

Figure 1: Four regions. $\Omega_{i}, i=1,2,3,4$, isolated. Emissions (left); Steady-state pollution level (right)

Both examples consider four regions $(J=4)$, each region $i$ controlling the averaged emissions over region $\Omega_{i}$. As Figures 1 and 2 show in this spatial configuration region 1 shares boundary with region 2 and the exterior of the whole region under consideration, $\Omega$; region 2 shares boundary with the other three regions; and regions 3 and 4 are completely symmetric with respect to their geographical position, they share a common boundary and a boundary with region 2 as well as with the exterior of the whole region $\Omega$. It is worth noting that this example is genuinely two dimensional in the sense that the particular spatial configuration showed in this example cannot be reproduced in a one-dimensional setting.

This example can represent a groundwater reservoir or a lake shared by four neighbouring regions. In the case of the groundwater reservoir pesticides or other contaminants (for example, phosphore) as a by-product of the agricultural activities in these regions pollute the reservoir. In the case of the lake, waste-water as a by-product of economic activities located in the regions pollutes the lake.

In the first example these four regions $\Omega_{1}, \ldots, \Omega_{4}$ are isolated from outside, in the sense that there is no flux of pollution neither entering nor exiting the geographical space formed by the four regions $\left(b_{i 0}=0, i=1, \ldots, 4\right)$. The groundwater reservoir or the lake is isolated from other sources or sinks of pollution. Figure 1 shows the equilibrium emission rates (left) and steady-state pollution level (right) for this example. As this figure shows region 3 and 4 emit at an identical rate, and hence, their steady-state pollution levels are identical too, because there is no difference 
between these two regions from a spatial (geographical) point of view. Figure 1 clearly illustrates the geographical advantage of region 2. Because this region has three neighbours to which the pollution can flow, along the equilibrium strategy it can emit at a rate clearly greater than that of regions 3 and 4 (Figure 1 left), but the steady-state level of the stock of pollution is similar for all these three regions (Figure 1 right). The region emitting at the lowest emission rate (region 1) attains the lowest stock of pollution in the long run.
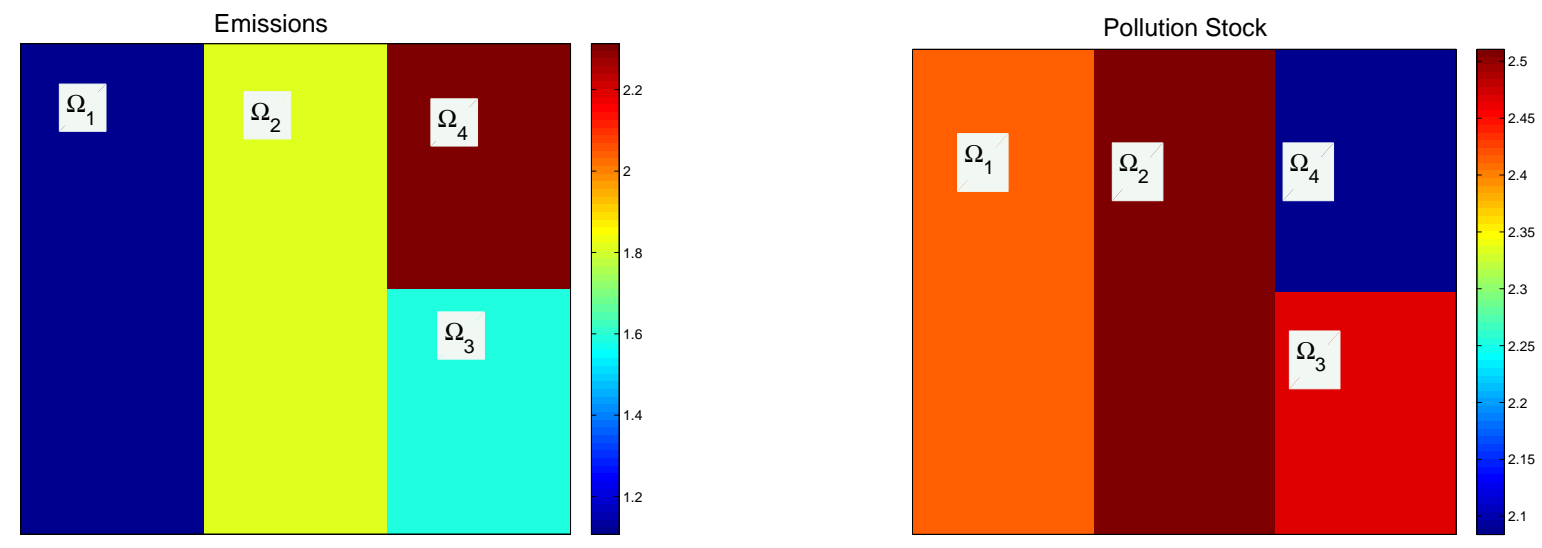

Figure 2: Four regions. $\Omega_{i}, i=1,2,3$, isolated, $\Omega_{4}$ has a clean neighbour. Emissions (left); Steady-state pollution level (right)

The second example is quite similar to the previous one, but presents a main difference. Unlike the previous example regions 1,2 and 3 are isolated from outside $\left(b_{10}=0\right)$, while region 4 shares a boundary with the exterior of the whole region $\Omega$ which is assumed to have a lower concentration of pollutants than region 4. This external region acts as a sink of pollution. In particular, as an extreme case, here we assume that the pollution stock in this additional region is zero $\left(q_{40}=0\right)$. With this geographical configuration there is a flux of pollution from region 4 to the pollution sink, but not in the opposite direction. Using the example of the aquifer, this external region could be a part of the aquifer that is not affected by human activities and shares boundary with region 4 . The size of the clean part of the aquifer is large enough to absorb pollutants with negligible increment of the concentration of pollutants.

As Figure 2 shows the region emitting at the highest level (region 4) presents the lowest stock of pollution in the long run. This in principle counterintuitive behavior comes as a result that there is a flow of pollution from region 4 to outside $\Omega$ while no flow is entering region 4 from outside $\Omega$. Because the stock of pollution is assumed to be zero outside $\Omega$, there is an important flow of pollution exiting region 4 towards the exterior of the whole region $\Omega$, allowing region 4 considerably increase its emissions in comparison with those of the other regions that because of their geographical positions only can "exchange" pollution concentration with the other regions in $\Omega$.

Figure 2 also shows that the regions closest to region 4 (regions 2 and 3) also benefit from the geographical position of the latter and their emission rates are greater than those of region 1 more distant from region 4. However, the steady-state values of the stock of pollution in regions 1,2 and 3 are quite similar, despite the fact that region 1 emits at a much softer level than the other regions. The sole reason for this behaviour is that region 1 only can exchange pollution with region 
2, while the other regions have two or three neighbours which can be the recipients of the flow of pollution.

It is worth noting that the somehow counterintuitive result "the higher the emissions, the lower the pollution stock" is due exclusively to the inclusion of the spatial aspect in the model at hand, stressing the importance of taking into account this aspect. This result cannot be reproduced in

a transboundary pollution dynamic game with symmetric players when the spatial transport of pollution is neglected.

These two numerical illustrations clearly show the impact of the strategic and spatially dynamic behaviour of the agents on the design of the environmental policies. Although the linear-state structure of the differential game prescribes equilibrium strategies which are constant over time, these two examples illustrate that the equilibrium strategies are very different depending on the geographical position of the players.

\section{Spatial vs. non-spatial model in a non-cooperative framework}

This section compares the equilibrium environmental policies of the spatial transboundary pollution problem stated in the previous sections which takes into account the spatial context with those equilibrium emission rates obtained as the optimal solution of a dynamic game that ignores the spatial transport phenomena. In order to simplify as much as possible this comparison we restrict to the two-player case. Although this assumption could be seen to be restrictive it allows us to easily characterize the equilibrium emission and investment rates of both differential games and present clear-cut results from the comparison.

Next proposition completely characterizes the feedback Nash equilibrium and the discounted net welfare of each player in the case of a 2-player differential game.

Proposition 2. The equilibrium emission and investment rates of the 2-player differential game defined by (10), (11) and (12) are constant and given by:

$$
I_{i}=\frac{\beta_{i}}{c_{i}\left(\mu_{i}+\rho\right)}, \quad e_{i}=\frac{\left(d_{j}+\rho\right) b+\left(b+d_{j}+\rho\right)\left(d_{i}+\rho\right)}{\eta_{i}\left(b+d_{j}+\rho\right) \varphi_{i}}, \quad i=1,2, i \neq j,
$$

where $b=b_{i j}=b_{j i}$ for $i \neq j$.

The discounted net welfare of player $i$ is given by:

$$
V_{i}\left(K_{i}, p_{i}, p_{j}\right)=M_{i, i} K_{i}+R_{i, i} p_{i}+R_{i, j} p_{j}+X_{i}
$$


where

$$
\begin{aligned}
M_{i, i} & =\frac{\beta_{i}}{\mu_{i}+\rho}, \quad R_{i, i}=-\frac{\varphi_{i}\left(b+d_{j}+\rho\right)}{\left(d_{i}+\rho\right)\left(b+d_{j}+\rho\right)+b\left(d_{j}+\rho\right)} \\
R_{i, j} & =-\frac{b \varphi_{i}}{\left(d_{i}+\rho\right)\left(b+d_{j}+\rho\right)+b\left(d_{j}+\rho\right)}, \quad X_{i}=-\frac{1}{2 c_{i} \rho\left(b+d_{i}+\rho\right) \varphi_{j}}\left(X_{i 1}+X_{i 2}\right), \\
X_{i 1} & =\frac{2 c_{i}}{\left(d_{i}+\rho\right)\left(b+d_{j}+\rho\right)+b\left(d_{j}+\rho\right)}\left[\left(b+d_{i}+\rho\right)\left(b+d_{j}+\rho\right)\left(d_{i}+\rho+q_{i} d_{i} \varphi_{i}\right) \varphi_{j}\right. \\
& \left.+b\left[b\left(d_{i}+\rho\right) \varphi_{i}+\left(b+d_{i}+\rho\right)\left(\left(\rho+d_{j}\right)\left(\varphi_{i}+\varphi_{j}\right)+d_{j} q_{i} \varphi_{i} \varphi_{j}\right)\right]\right] \\
X_{i 2} & =-\left(b+d_{i}+\rho\right) \varphi_{j}\left(\frac{\beta_{i}^{2}}{\left(\mu_{i}+\rho\right)^{2}}+2 c_{i} \log \left(\frac{\left(d_{i}+\rho\right)\left(b+d_{j}+\rho\right)+b\left(d_{j}+\rho\right)}{\eta_{i}\left(b+d_{j}+\rho\right) \varphi_{i}}\right)\right),
\end{aligned}
$$

for $i, j=1,2, i \neq j$.

The steady-state levels of clean technology and pollution stocks are given by:

$$
\begin{aligned}
K_{i}^{S S} & =\frac{\beta_{i}}{c_{i} \mu_{i}\left(\mu_{i}+\rho\right)}, \\
p_{i}^{S S} & =\frac{N u m p_{i}^{S S}}{\left(d_{i} d_{j}+b\left(d_{i}+d_{j}\right)\right)\left(b+d_{i}+\rho\right)\left(b+d_{j}+\rho\right) \varphi_{i} \varphi_{j}}, \\
N u m p_{i}^{S S} & =\left(b+d_{j}\right)\left(b+d_{i}+\rho\right)\left(b\left(d_{j}+\rho\right)+\left(b+d_{j}+\rho\right)\left(d_{i}+\rho+q_{i} d_{i} \varphi_{i}\right)\right) \varphi_{j} \\
& +b\left(b+d_{j}+\rho\right) \varphi_{i}\left(b\left(d_{i}+\rho\right)+\left(b+d_{i}+\rho\right)\left(d_{j}+\rho+q_{j} d_{j} \varphi_{j}\right)\right),
\end{aligned}
$$

for $i, j=1,2, i \neq j$, where the superscript $S S$ stands for steady-state levels and $q_{k}$ is the aggregation of the boundary data defined in (12). The steady-state equilibrium is globally asymptotically stable.

As expected the discounted net welfare of each region depends positively on its own stock of clean technology and negatively on both stocks of pollution. As a result of the linearity of the value function, the equilibrium emission and investment rates are constant with respect to the state variables and hence they are constant over time. It is worth noting that $b$ and $q_{i}$, two of the most significant parameters of the spatial diffusion model, affect the equilibrium emission rate and the steady-state levels of the stock of pollution. Next corollary collects the results of the sensitivity analysis with respect to these two main parameters.

Corollary 1. 1. The equilibrium emission rate, $e_{i}$, increases as the diffusion parameter $b$ increases.

2. The steady-state levels of the stocks of pollution, $p_{i}^{S S} i=1,2$, increase as parameters $q_{i}$ and $q_{j}$ increase.

3. In a completely symmetric setting, the steady-state levels of the stocks of pollution $p_{i}^{S S} i=1,2$, increase as the diffusion parameter $b$ increases.

The first item establishes that the higher $b$, the faster the stock of pollution diffuses from one region to another, and as a result, the region takes advantage of the opportunity to emit at a higher rate. A greater $q_{k}$ indicates a greater stock of pollution in the neighbourhood of the region under consideration, and by the Fick's law this region would be the recipient of pollution coming from 
outside until the stock of pollution inside and outside the region stabilizes. As a consequence, the result in the second item follows. The third item shows that for the completely symmetric scenario, $d_{i}=d_{j}=d, q_{i}=q_{j}=q, \varphi_{i}=\varphi_{j}=\varphi$ the effect of the diffusion parameter $b$ on the steady-state of the stock of pollution is clearly positive.

However, in general the effect of the diffusion parameter $b$ on the steady-state of the stock of pollution is unclear as the following corollary shows.

Corollary 2. Under assumptions $d_{i}=d_{j}=d, q_{i}=q_{j}=q$, the following results apply:

1. If $\varphi_{i} \geq \varphi_{j}$, then the steady-state level of the stock of pollution in region $i, p_{i}^{S S}$, increases as the diffusion parameter $b$ increases.

2. If $\varphi_{i}<\varphi_{j}$, then

(a) for large $b, p_{i}^{S S}$, increases with $b$;

(b) for small $b, p_{i}^{S S}$, decreases with $b$ if and only if $\varphi_{i}<\frac{\rho}{d+\rho} \varphi_{j}$.

From Corollary 1 any increase in the diffusion parameter $b$ leads to an increase of the equilibrium emission rates in both regions. One should expect that these greater emissions rates would always imply a greater steady-state level of the pollution stock in both regions. Corollary 2 shows that this is not always the case. Although the total average long-run pollution stock $\left(p_{i}^{S S}+p_{j}^{S S}\right) / 2$ always increases with $b$, it can be the case that for small $b, p_{i}^{S S}$ decreases with $b$ if $\varphi_{i}<\frac{\rho}{d+\rho} \varphi_{j}$. Under this last condition, and for fixed values of the other parameters, the emission rate in region $j, e_{j}$, would be small compared to $e_{i}$. Hence, the global effect on $p_{i}^{S S}$ of a greater $b$ due to the diffusion process could be a lower $p_{i}^{S S}$.

In order to evaluate the impact of the introduction of the spatial aspect in the economicenvironmental model we briefly describe the differential game proposed by Jørgensen \& Zaccour (2001) where the spatial dimension is ignored. For brevity we refer to this model as the non-spatial model and we add a tilde on the variables and parameters linked to the environmental part of the model to distinguish them from those used in the spatial model. We have used identical notation in both models for the variables and parameters related to the investment in clean technology and to the stock of clean technology because we have not included any spatial aspect in this part of the model.

Region $i$ in the non-spatial model chooses its control variables, the emission and investment rates, $\widetilde{e}_{i}$ and $I_{i}$ in order to maximize

$$
\widetilde{J}_{i}\left(\widetilde{e}_{i}, I_{i}, \widetilde{P}_{0}, K_{i 0}\right)=\int_{0}^{\infty} e^{-\rho t}\left(\log \left(\widetilde{e}_{i}\right)+\beta_{i} K_{i}-\frac{1}{2} c_{i} I_{i}^{2}-\widetilde{\varphi}_{i} \widetilde{P}\right) \mathrm{d} t
$$

subject to the dynamics of its own stock of clean technology given by (10) and of the stock of pollution which evolves over time according to:

$$
\dot{\widetilde{P}}=\widetilde{\eta}_{1} \widetilde{e}_{1}+\widetilde{\eta}_{2} \widetilde{e}_{2}-\widetilde{d P}, \quad \widetilde{P}(0)=\widetilde{P}_{0}
$$

For comparison purposes next proposition characterizes the feedback Nash equilibrium and the discounted net welfare of each player of the non-spatial 2-player differential game. 
Proposition 3. The equilibrium emission and investment rates of the 2-player differential game defined by (10), (22) and (23) are constant and given by:

$$
I_{i}=\frac{\beta_{i}}{c_{i}\left(\mu_{i}+\rho\right)}, \quad \widetilde{e}_{i}=\frac{\rho+\widetilde{d}}{\widetilde{\eta}_{i} \widetilde{\varphi}_{i}}, \quad i=1,2, i \neq j .
$$

The discounted net welfare of player $i$ is given by:

$$
\widetilde{V}_{i}\left(K_{i}, \tilde{P}\right)=M_{i} K_{i}+\widetilde{R}_{i} \widetilde{P}+\widetilde{X}_{i}
$$

where

$$
\begin{aligned}
M_{i} & =\frac{\beta_{i}}{\mu_{i}+\rho}, \quad \widetilde{R}_{i}=-\frac{\widetilde{\varphi}_{i}}{\rho+\widetilde{d}}, \\
\widetilde{X}_{i} & =\frac{1}{2 \rho}\left(\frac{\beta_{i}^{2}}{c_{i}\left(\mu_{i}+\rho\right)^{2}}-\frac{2\left(\widetilde{\varphi}_{i}+\widetilde{\varphi}_{j}\right)}{\widetilde{\varphi}_{j}}+2 \log \left(\frac{\rho+\widetilde{d}}{\widetilde{\eta}_{i} \widetilde{\varphi}_{i}}\right)\right),
\end{aligned}
$$

for $i=1,2, i \neq j$.

The steady-state levels of clean technology and pollution stocks are given by:

$$
K_{i}^{S S}=\frac{\beta_{i}}{c_{i} \mu_{i}\left(\mu_{i}+\rho\right)}, \quad \widetilde{P}^{S S}=\frac{(\rho+\widetilde{d})\left(\widetilde{\varphi}_{i}+\widetilde{\varphi}_{j}\right)}{\widetilde{d} \widetilde{\varphi}_{i} \widetilde{\varphi}_{j}},
$$

for $i=1,2, i \neq j$. The steady-state equilibrium is globally asymptotically stable.

From the comparison of both models it is clear that the equilibrium investment rates and the steady-state levels of the stock of clean technology are identical. This result is completely expected because the spatial differential game has only incorporated the spatial aspect in the pollution dimension and has disregarded this aspect in the stock of clean technology. Hence, both models are identical as far as the equilibrium investment rates and the steady-state levels of the stock of clean technology are concerned. Therefore, from now on we focus on the comparison of the environmental variables, equilibrium emissions rates and steady-state levels of the stocks of pollution, as well as the discounted net welfare.

Next proposition shows one of the main results of our study because it establishes that our spatial model is a generalization of the non-spatial model in the sense that the behaviour of the environmental variables at the equilibrium in the non-spatial setting can be reproduced as a limit case of the spatial setting. In particular, this link is obtained when the parameter describing how pollution diffuses among regions increases unboundedly. Specifically, when the diffusion parameter tends to infinity, the mixing of the stocks of pollution in both regions is instantaneous, which is the main hypothesis in the non-spatial differential game.

In order to have comparable models from now on we assume that the following hypotheses are satisfied:

Hypotheses $(\mathbf{H})$. Parameters $\rho, \mu_{i}, \beta_{i}, c_{i}, i=1,2$ are identical in the differential games described 
by (10), (11) and (12), and (10), (22) and (23), respectively, and

$$
\eta_{1}=\eta_{2}=2 \tilde{\eta}_{1}=2 \tilde{\eta}_{2} ; \quad d_{1}=d_{2}=\tilde{d} ; \quad b_{10}=b_{20}=0 ; \quad \varphi_{i}=\tilde{\varphi}_{i}, i=1,2 .
$$

We remark that hypotheses $\mathbf{H}$ allow the two models to be comparable. More explicitly the hypothesis $d_{1}=d_{2}=\tilde{d}$ simply means that the rate of natural decay of the pollution stock is identical in both regions $\Omega_{1}$ and $\Omega_{2}$ and corresponds to the rate of decay of the only region in the model defined by (10), (22) and (23). Furthermore, in the non-spatial model the region under consideration is supposed by definition to be isolated from the exterior. This is exactly the meaning of hypothesis $b_{10}=b_{20}=0$ (see (9)). It is more interesting the meaning of $\eta_{1}=\eta_{2}=2 \tilde{\eta}_{1}=2 \tilde{\eta}_{2}$. First, we are in both cases in a symmetric scenario $\left(\eta_{1}=\eta_{2}\right.$ and $\left.\tilde{\eta}_{1}=\tilde{\eta}_{2}\right)$; and, second, in the non-spatial dynamics (23) the emissions of both players instantaneously affect the whole domain $\Omega$ under consideration. Conversely, in the spatial dynamics (12) the emissions of player $i$ only affect region $\Omega_{i}$. The size of $\Omega_{i}$ is supposed, if the rest of parameters are identical for both players, to be half the total area of domain $\Omega$. So, the effect of emissions on the rate of change of the pollution stock in domain $\Omega$ are comparable for $\eta_{1}=\eta_{2}=2 \tilde{\eta}_{1}=2 \tilde{\eta}_{2}$.

Proposition 4. Under hypotheses $\mathbf{H}$, as the diffusion parameter $b$ tends to infinity, then

i) the equilibrium emission rate $e_{i}$ converges to $\widetilde{e}_{i}$;

ii) the average of the steady-state levels of the pollution stocks $\frac{p_{i}^{S S}+p_{j}^{S S}}{2}$ converges to $\widetilde{P}^{S S}$. Furthermore, if both regions are completely symmetric, then $p_{i}^{S S}=p_{j}^{S S}=\widetilde{P}^{S S}$.

The results in Proposition 4 show that even for this simple model where equilibrium strategies are constant, our formulation of the spatial model is a generalization of the non-spatial version of the model capturing the main ingredients of the spatial dynamics. The gaps above tend to zero as the diffusion parameter $b$ goes to infinity, which represents an instantaneous mix of the pollution stocks. However, the largest gaps arise when the diffusion parameter is zero, which can be viewed as the extreme case where there is not diffusion of pollution from one region to another.

In view of these results and in order to evaluate the effect of the diffusion parameter on the equilibrium emissions rates and steady-state levels of the stocks of pollution, next proposition compares the environmental variables under hypotheses $\mathbf{H}$.

Proposition 5. Under hypotheses $\mathbf{H}$, for any finite value of the diffusion parameter $b$ :

i) the equilibrium emission rate $e_{i}$ satisfies:

$$
\frac{\widetilde{e}_{i}}{2} \leq e_{i} \leq \widetilde{e}_{i}
$$

ii) the average of the steady-state levels of the pollution stock satisfies

$$
\frac{\widetilde{P}^{S S}}{2} \leq \frac{p_{i}^{S S}+p_{j}^{S S}}{2} \leq \widetilde{P}^{S S}
$$

When $b=0$, there is not diffusion of pollution from one region to another. All the accumulated emissions of regions $i$ remain in $\Omega_{i}$, and there is no flux of pollution coming from outside. Region 
$\Omega_{i}$ is isolated from $\Omega_{j}$. On the contrary, as the diffusion parameter $b$ goes to infinity, there is a perfect mixing of the pollution stocks. In the completely symmetric scenario both regions have identical size, which is half of the total area of domain $\Omega$. Therefore, under perfect mixing half of the emission of region $i$ instantaneously flows to region $j$. As a consequence, region $i$ doubles the emission rate with respect to the case $b=0$.

When $b$ is positive and finite, the optimal amount of emissions in $\Omega_{i}$ depends on how fast part of the stock of pollution in region $i$ is transferred to region $j$ via the diffusion process. The greater parameter $b$, the greater the speed of diffusion. Note that the units of $b$ are the inverse of the units of time.

Finally, we assess the impact of the diffusion parameter on the discounted net welfare. Next proposition compares the discounted net welfare for both spatial and non-spatial dynamic games.

Proposition 6. Under hypotheses $\mathbf{H}$, then,

i) As the diffusion parameter $b$ tends to infinity, the long-run discounted net welfare of player $i$ in the spatial model, $V_{i}\left(K_{i}^{S S}, p_{i}^{S S}, p_{j}^{S S}\right)$, converges to the long-run discounted net welfare of player $i$ in the non-spatial model, $\widetilde{V}_{i}\left(K_{i}^{S S}, \widetilde{P}^{S S}\right)$.

ii) For the completely symmetric scenario $\varphi_{i}=\varphi_{j}=\varphi$, and for any finite value of the diffusion parameter $b, V_{i}\left(K_{i}^{S S}, p_{i}^{S S}, p_{j}^{S S}\right)$, is always greater than $\widetilde{V}_{i}\left(K_{i}^{S S}, \widetilde{P}^{S S}\right)$.

The second item in Proposition 6 shows that for the completely symmetric scenario, the effect of taking into account the spatial aspect of the problem on the long-run discounted net welfare is clearly positive.

However, in general this effect is unclear as the following corollary shows.

Corollary 3. 1. For any fixed value of $\varphi_{j}$, if the environmental damage cost parameter of player $i, \varphi_{i}$, is large enough, then $V_{i}\left(K_{i}^{S S}, p_{i}^{S S}, p_{j}^{S S}\right)$ is greater than $\widetilde{V}_{i}\left(K_{i}^{S S}, \widetilde{P}^{S S}\right)$.

2. For any fixed value of $\varphi_{i}$, if the environmental damage cost parameter of player $j, \varphi_{j}$ and $d$ are large enough, then $V_{i}\left(K_{i}^{S S}, p_{i}^{S S}, p_{j}^{S S}\right)$ is lower than $\widetilde{V}_{i}\left(K_{i}^{S S}, \widetilde{P}^{S S}\right)$.

If the regions behave myopically, disregarding the spatial dimension of the problem, then they are considering that the diffusion parameter $b$, that measures the speed at which pollution stocks are mixed, is infinity. In other words, the regions are assuming that there is an instantaneous mixing of the pollution stocks. This is the standard hypothesis in the literature which does not consider the spatial dimension. From Proposition 4 when the regions do not take into account their geographical positions when deciding their optimal emission levels, both regions will be emitting $\widetilde{e}_{i}$, above their optimal equilibrium level $e_{i},\left(e_{i}<\widetilde{e}_{i}\right)$. These spatially myopic optimal levels of emissions lead to a long-run pollution stock $\widetilde{P}^{S S}$ greater than the average of both steady-state pollution stocks when the regions acknowledge the spatial aspect when taking their optimal strategic decisions $\frac{p_{i}^{S S}+p_{j}^{S S}}{2}$. The myopic optimal levels of emissions can be explained through what could be considered a freeriding effect. In the infinite diffusion case (spatially myopic case, $b$ converging to infinity), there is perfect mixing of the pollution stocks, and hence, this implies that each region can emit at a greater level because it knows that the effect of its own emissions is instantaneously shared with the other region. 
On the contrary, as shown in Proposition 5 in the finite diffusion case (spatially non-myopic case, $b$ finite), without perfect mixing of the pollution stocks, each region takes into account that it is affected by its own emissions for a longer period of time; in the sense that although asymptotically the pollution stocks in both regions tend to equilibrate, in every finite time interval the accumulated pollution due to its own emissions is greater than in the infinite diffusion case. The greater the diffusion parameter, the shorter the time interval from which the effect of diffusion is negligible.

If the regions behave myopically with respect to their spatial position there are two opposite forces affecting their long-run welfares. On the one hand, a greater emission level rises up the revenues related to production described by $\log \left(\widetilde{e}_{i}\right)$. On the other hand, a greater long-run pollution stock $\widetilde{P}^{S S}$ pushes up the environmental damages represented by $\widetilde{\varphi} \widetilde{P}^{S S}$. Hence, at a first glance, the global effect on long-run welfares is unclear. However, if both regions are completely symmetric the second effect is stronger and more than overcompensates the first effect. Consequently, the symmetric regions are underestimating their long-run welfares with respect to their welfares when the regions acknowledge the geographical aspect of the problem (Proposition 6 item ii)). For asymmetric regions and depending on the value of the environmental damage cost parameter each region could over or under-estimate their long-run welfares (Corollary 3 ).

The managerial implications are that the geographical/spatial aspects are essential ingredients when determining the equilibrium values of the emission levels. The spatially non-myopic behavior prescribes lower equilibrium emission rates, and consequently, a lower global pollution stock. Interestingly, this is compatible with greater welfares in the long run.

\section{Spatial vs. non-spatial model in a cooperative framework}

In order to assess the impact of the spatial aspects on the definition of the emissions and investment policies in a cooperative setting, in this section we focus on the comparison of the cooperative strategies of the transboundary pollution dynamic games with and without spatial effects. For the ease of presentation and as in the previous section we restrict ourselves to the twoplayer case. However, the main conclusions derived along this section can be easily extended to the more general case where the number of players is greater than 2. Next proposition characterizes the cooperative solution obtained solving an optimal control problem where the unique decision-maker chooses both regions' emission policies as well as the investment in the stock of clean technology in order to maximize the joint welfare of both regions

$$
J^{c}\left(e_{1}, e_{2}, I_{1}, I_{2}, \boldsymbol{p}^{0}, K_{10}, K_{20}\right)=\int_{0}^{\infty} \sum_{i=1}^{2} e^{-\rho t}\left(\log \left(e_{i}\right)+\beta_{i} K_{i}-\frac{1}{2} c_{i} I_{i}^{2}-\varphi_{i} p_{i}\right) \mathrm{d} t,
$$

subject to the dynamics of both stocks of clean technology given by (10) and of the aggregated stock of pollution in each region defined by (12).

Proposition 7. The cooperative emission and investment rates of the optimal control problem defined by (10), (12) and (25) are constant and given by:

$$
I_{i}^{c}=\frac{\beta_{i}}{c_{i}\left(\mu_{i}+\rho\right)}, \quad e_{i}^{c}=\frac{\left(d_{j}+\rho\right) b+\left(b+d_{j}+\rho\right)\left(d_{i}+\rho\right)}{\eta_{i}\left(\left(b+d_{j}+\rho\right) \varphi_{i}+b \varphi_{j}\right)}, \quad i=1,2, i \neq j,
$$


where $b=b_{i j}=b_{j i}$ for $i \neq j$ and the superscript c stands for cooperative.

The optimal cooperative discounted net welfare is given by:

$$
V^{c}\left(K_{i}, K_{j}, p_{i}, p_{j}\right)=M^{c} K_{i}+N^{c} K_{j}+R^{c} p_{i}+T^{c} p_{j}+X^{c}
$$

where

$$
\begin{aligned}
M^{c} & =M_{i, i}=\frac{\beta_{i}}{\mu_{i}+\rho}, \quad N^{c}=M_{j, j}=\frac{\beta_{j}}{\mu_{j}+\rho}, \\
R^{c} & =-\frac{\varphi_{i}\left(b+d_{j}+\rho\right)+b \varphi_{j}}{\left(d_{i}+\rho\right)\left(b+d_{j}+\rho\right)+b\left(d_{j}+\rho\right)}, T^{c}=-\frac{\varphi_{j}\left(b+d_{i}+\rho\right)+b \varphi_{i}}{\left(d_{i}+\rho\right)\left(b+d_{j}+\rho\right)+b\left(d_{j}+\rho\right)}, \\
X^{c} & =\frac{1}{2 \rho}\left(X_{1}^{c}+X_{2}^{c}\right), \\
X_{1}^{c} & =-2-\frac{d_{i} q_{i}\left(\left(d_{j}+\rho\right) \varphi_{i}+b\left(\varphi_{i}+\varphi_{j}\right)\right)+d_{j} q_{j}\left(\left(d_{i}+\rho\right) \varphi_{j}+b\left(\varphi_{i}+\varphi_{j}\right)\right)}{\left(d_{i}+\rho\right)\left(d_{j}+\rho\right)+b\left(d_{i}+d_{j}+2 \rho\right)}, \\
X_{2}^{c} & =\frac{\beta_{i}^{2}}{c_{i}\left(\mu_{i}+\rho\right)^{2}}+\frac{\beta_{j}^{2}}{c_{j}\left(\mu_{j}+\rho\right)^{2}}+2 \log \left(\frac{\left(b\left(d_{i}+\rho\right)+\left(b+d_{i}+\rho\right)\left(d_{j}+\rho\right)\right)^{2}}{\eta_{i} \eta_{j}\left(\left(b+d_{j}+\rho\right) \varphi_{i}+b \varphi_{j}\right)\left(\left(b+d_{i}+\rho\right) \varphi_{j}+b \varphi_{i}\right)}\right),
\end{aligned}
$$

for $i, j=1,2, i \neq j$.

The cooperative steady-state levels of clean technology and pollution stocks are given by:

$$
\begin{gathered}
K_{i}^{S S c}=\frac{\beta_{i}}{c_{i} \mu_{i}\left(\mu_{i}+\rho\right)}, \quad p_{i}^{S S c}=\frac{N u m p_{i}^{S S c}}{d_{i} d_{j}+b\left(d_{i}+d_{j}\right)}, \\
N u m p_{i}^{S S c}=b q_{j} d_{j}+q_{i} d_{i}\left(b+d_{j}\right) \\
+\left(\left(d_{i}+\rho\right)\left(d_{j}+\rho\right)+b\left(d_{i}+d_{j}+2 \rho\right)\right)\left(\frac{b}{\left(b+d_{i}+\rho\right) \varphi_{j}+b \varphi_{i}}+\frac{b+d_{j}}{b \varphi_{j}+\left(b+d_{j}+\rho\right) \varphi_{i}}\right),
\end{gathered}
$$

for $i, j=1,2, i \neq j$. The steady-state equilibrium is globally asymptotically stable.

Similarly, in the cooperative formulation of the non-spatial model the unique decision-maker chooses both regions' emission policies as well as the investment in the stock of clean technology in order to maximize the joint welfare of both regions

$$
\widetilde{J}^{c}\left(\widetilde{e}_{1}, \widetilde{e}_{2}, I_{1}, I_{2}, \widetilde{P}_{0}, K_{10}, K_{20}\right)=\int_{0}^{\infty} \sum_{i=1}^{2} e^{-\rho t}\left(\log \left(\widetilde{e}_{i}\right)+\beta_{i} K_{i}-\frac{1}{2} c_{i} I_{i}^{2}-\widetilde{\varphi}_{i} \widetilde{P}\right) \mathrm{d} t
$$

subject to the dynamics of both stocks of clean technology given by (10) and of the stock of pollution defined by (23). Next proposition characterizes the cooperative solution of this optimal control problem.

Proposition 8. The cooperative emission and investment rates of the optimal control problem defined by (10), (23) and (27) are constant and given by:

$$
I_{i}^{c}=\frac{\beta_{i}}{c_{i}\left(\mu_{i}+\rho\right)}, \quad \widetilde{e}_{i}^{c}=\frac{\rho+\widetilde{d}}{\widetilde{\eta}_{i}\left(\widetilde{\varphi}_{i}+\widetilde{\varphi}_{j}\right)}, \quad i=1,2, i \neq j .
$$


The optimal cooperative discounted net welfare is given by:

$$
\widetilde{V}^{c}\left(K_{i}, K_{j}, \tilde{P}\right)=M^{c} K_{i}++N^{c} K_{j}+\widetilde{R}^{c} \widetilde{P}+\widetilde{X}^{c}
$$

where

$$
\begin{aligned}
M^{c} & =M_{i, i}=\frac{\beta_{i}}{\mu_{i}+\rho}, \quad N^{c}=M_{j, j}=\frac{\beta_{j}}{\mu_{j}+\rho}, \quad \widetilde{R}^{c}=-\frac{\widetilde{\varphi}_{i}+\widetilde{\varphi}_{j}}{\rho+\widetilde{d}}, \\
\widetilde{X}^{c} & =\frac{1}{2 \rho}\left(\frac{\beta_{i}^{2}}{c_{i}\left(\mu_{i}+\rho\right)^{2}}+\frac{\beta_{j}^{2}}{c_{j}\left(\mu_{j}+\rho\right)^{2}}-4+2 \log \left(\frac{(\widetilde{d}+\rho)^{2}}{\widetilde{\eta}_{i} \widetilde{\eta}_{j}\left(\widetilde{\varphi}_{i}+\widetilde{\varphi}_{j}\right)}\right)\right),
\end{aligned}
$$

for $i=1,2, i \neq j$.

The cooperative steady-state levels of clean technology and pollution stocks are given by:

$$
K_{i}^{S S c}=\frac{\beta_{i}}{c_{i} \mu_{i}\left(\mu_{i}+\rho\right)}, \quad \widetilde{P}^{S S c}=\frac{2(\rho+\widetilde{d})}{\widetilde{d}\left(\widetilde{\varphi}_{i}+\widetilde{\varphi}_{j}\right)},
$$

for $i=1,2, i \neq j$. The steady-state equilibrium is globally asymptotically stable.

As one can note, the cooperative investment rates for both optimization problems are the same as the equilibrium investment strategies obtained in the non-cooperative frameworks. This result implies that the Nash equilibrium investment strategies are Pareto optimal. This comes as a result of the structure of the model where there is no interaction between the investment decisions of the players. The interaction between the emission decisions of the players through their effect on the accumulation of the pollution stock(s) implies that the cooperative emission rates are different from the equilibrium emission strategies obtained in the non-cooperative settings. It can be easily shown that, as expected, the emission levels are lower under cooperation (both for the spatial and non-spatial differential games). The difference between cooperative and non-cooperative emission rates lies in the fact that in the non-cooperative setting each region (player) takes into account only his marginal damage cost $\left(\varphi_{i}\right.$ or $\widetilde{\varphi}_{i}$ for the spatial and non-spatial models, respectively), while in the cooperative setting the decision-maker takes into account both players marginal damage costs $\left(\varphi_{i}, \varphi_{j}\right.$ or $\left.\widetilde{\varphi}_{i}, \widetilde{\varphi}_{j}\right)$. Because the emission levels are lower under cooperation, the steady-state level of each stock of pollution is lower under cooperation too.

Next three propositions follow the same patterns as Propositions 4, 5 and 6 but now for the cooperative setting. Similarly to Proposition 4 and under the same assumptions on the model parameters next we show that also for the cooperative framework our spatial model generalizes the non-spatial model: As the diffusion parameter tends to infinity, the environmental variables at the equilibrium in the spatial model converge to those variables in the non-spatial setting.

Proposition 9. Under hypotheses $\mathbf{H}$, as the diffusion parameter b tends to infinity,

i) the cooperative emission rate $e_{i}^{c}$ converges to $\widetilde{e}_{i}^{c}$;

ii) the average of the cooperative steady-state levels of the pollution stocks $\frac{p_{i}^{S S c}+p_{j}^{S S c}}{2}$ converges to $\widetilde{P}^{S S c}$. Furthermore, if both regions are completely symmetric, then $p_{i}^{S S c}=p_{j}^{S S c}=\widetilde{P}^{S S c}$. 
As in the non-cooperative setting, the gaps above tend to zero as the diffusion parameter $b$ goes to infinity (instantaneous mix of the stocks of pollution) and the gaps increase as the diffusion parameter tends to zero (the stock of pollution does not spread from one region to another).

Next proposition compares the cooperative emissions rates and cooperative steady-state levels of the stocks of pollution for the spatial and non-spatial models under hypotheses $\mathbf{H}$, in order to evaluate the effect of the diffusion parameter on the environmental variables.

Proposition 10. Under hypotheses $\mathbf{H}$, for any finite value of the diffusion parameter b:

i) the cooperative emission rate satisfies

$$
\min \left\{\widetilde{e}_{i}^{c}, \frac{\varphi_{i}+\varphi_{j}}{2 \varphi_{i}} \widetilde{e}_{i}^{c}\right\} \leq e_{i}^{c} \leq \max \left\{\widetilde{e}_{i}^{c}, \frac{\varphi_{i}+\varphi_{j}}{2 \varphi_{i}} \widetilde{e}_{i}^{c}\right\}
$$

ii) the average of the cooperative steady-state levels of the pollution stocks satisfies

$$
\widetilde{P}^{S S c} \leq \frac{p_{i}^{S S c}+p_{j}^{S S c}}{2} \leq \frac{\left(\varphi_{i}+\varphi_{j}\right)^{2}}{4 \varphi_{i} \varphi_{j}} \widetilde{P}^{S S c}
$$

iii) If the marginal environmental damage costs are identical for the two regions $\left(\varphi_{i}=\varphi_{j}\right)$, then $e_{i}^{c}=\widetilde{e}_{i}^{c}$, and consequently, $\frac{p_{i}^{S S c}+p_{j}^{S S c}}{2}=\widetilde{P}^{S S c}$.

It is worth noting that the lower and upper bounds of the equilibrium emission rate $e_{i}^{c}$ in item i) depend on how the marginal environmental damage costs of the regions compare. If $\varphi_{i}>\varphi_{j}$, then $e_{i}^{c}$ runs between $\frac{\varphi_{i}+\varphi_{j}}{2 \varphi_{i}} \widetilde{e}_{i}^{c}$ and $\widetilde{e}_{i}^{c}$, and the cooperative emission rate of region $i$ in the spatial model is always lower than the corresponding rate in the non-spatial model. However, if $\varphi_{i}<\varphi_{j}$, then $e_{i}^{c}$ runs between $\widetilde{e}_{i}^{c}$ and $\frac{\varphi_{i}+\varphi_{j}}{2 \varphi_{i}} \widetilde{e}_{i}^{c}$, and the cooperative emission rate of region $i$ in the spatial model is always greater than the corresponding rate in the non-spatial model.

Proposition 9 shows that if $b$ tends to infinity, the same underlying mechanism as in the noncooperative scenario is applicable.

When $b$ is zero, the regions are completely isolated and there is no interaction between the emission variables neither in the net benefit functions of each region nor in the dynamics of the pollution stocks. Therefore, the social planner will optimally choose the emission rate for each region identical to what each region would choose by itself in the non-cooperative framework, $e_{i}^{c}=e_{i}, i=1,2$. Let us note that this result is completely different from that obtained in the non-spatial setting, where cooperative emission rates are strictly lower than non-cooperative ones.

In the spatial cooperative framework for any finite value of the diffusion parameter $b$ if the environmental damage parameters are identical in both regions $\varphi_{i}=\varphi_{j}$, the social planner is indifferent about the location of emissions, and hence, the effect of diffusion disappears. The unique decision-maker equally values that the pollution stock lasts for a longer period in one region or in its neighbour, and what interests him is the average pollution (Proposition 10 item iii)).

If the environmental damage cost parameters are different, for example, $\varphi_{i}>\varphi_{j}$, the social planner values in a greater extent the environmental damage in region $\Omega_{i}$ than in region $\Omega_{j}$. For any fixed value of $b$, he will optimally decide to emit at a greater level in region $j$ than in region $i$ (Proposition 7). As parameter $b$ is increasing, the mixing of the pollution stocks becomes faster, and 
therefore, the optimal emission in region $\Omega_{i}$ could be greater, because part of the pollution stock in this region is diffused faster to $\Omega_{j}$, where the environmental damage is smaller. The opposite reasoning applies if $\varphi_{i}<\varphi_{j}$.

We can conclude that in the completely symmetric scenario the spatial effect disappears in the cooperative framework. However, as soon as the symmetry disappears, the spatial aspect plays an important role again.

Next proposition compares the long-run discounted net welfare for both spatial and non-spatial cooperative dynamic games.

Proposition 11. Under hypotheses $\mathbf{H}$, then,

i) As the diffusion parameter tends to infinity, the long-run cooperative discounted net welfare in the spatial model, $V^{c}\left(K_{i}^{S S c}, K_{j}^{S S c}, p_{i}^{S S c}, p_{j}^{S S c}\right)$, converges to the the corresponding value in the non-spatial model, $\widetilde{V}^{c}\left(K_{i}^{S S c}, K_{j}^{S C}, \widetilde{P}^{S S c}\right)$.

ii) For the completely symmetric scenario $\left(\varphi_{i}=\varphi_{j}=\varphi\right)$, and for any value of the diffusion parameter $V^{c}\left(K_{i}^{S S c}, K_{j}^{S S c}, p_{i}^{S S c}, p_{j}^{S S c}\right)$ and $\widetilde{V}^{c}\left(K_{i}^{S S c}, K_{j}^{S S c}, \widetilde{P}^{S S c}\right)$ are identical.

The second item in Proposition 11 shows that for the completely symmetric scenario, the effect of taking into account or disregarding the spatial aspect of the problem on the long-run discounted net welfare is null.

However, this effect could be positive or negative as the following corollary shows.

Corollary 4. 1. For any finite value of the diffusion parameter $b$, if $d \geq 2 \rho$, then $V^{c}\left(K_{i}^{S S c}, K_{j}^{S S c}, p_{i}^{S S c}, p_{j}^{S S c}\right)$ is greater than $\widetilde{V}^{c}\left(K_{i}^{S S c}, K_{j}^{S S c}, \widetilde{P}^{S S c}\right)$.

2. If $d<2 \rho$, then $V_{i}\left(K_{i}^{S S}, p_{i}^{S S}, p_{j}^{S S}\right)$ is greater (lower) than $\widetilde{V}_{i}\left(K_{i}^{S S}, \widetilde{P}^{S S}\right)$ for $b$ small (large).

Summarizing the results in this section, in the cooperative framework and if the regions are completely symmetric, the only decision-maker equally values the stocks of pollution of both regions. Hence, his decisions are identical regardless of whether the pollution stocks are or not instantaneously mixed. Because the decision maker values the pollution stocks equally in both regions, what matters is the average (Proposition 10 item iii). As a result the long-run welfare is the same regardless of whether or not the geographical dimension is taken into account (Proposition 11 item ii).

If the regions do not evaluate equally the environmental damage caused by pollution, the only decision-maker in the cooperative setting behaves strategically from a spatial point of view. If the only factor of asymmetry is the environmental damage cost (and all the other parameters remain unchanged) the optimal emission will be greater in the region with a lower environmental damage cost (Proposition 7). On the contrary, with a perfect mixing of the pollution stocks $(b$ converging to infinity), the optimal emission levels are identical among regions regardless of the environmental damage cost parameters (Proposition 8). Without a perfect mixing of the pollution stocks ( $b$ finite), the social planner will decide strategically under (over) emit in one region with respect to the perfect mixing scenario ( $b$ converging to infinity) when the environmental damage cost parameter is greater (lower) than in the other region (see (A.9)). The decision-maker is applying a sharing rule of emissions between the regions taking into account the free-riding effect previously described in the non-cooperative framework at the end of Section 4. 
The analysis above clearly shows the relevance of the spatial dimension in the optimal social planners decisions also in the cooperative framework.

There are two main implications of the previous analysis carried out in the cooperative framework. First, if both regions are completely symmetric, the optimal emission rates prescribed by the social planner will be identical regardless of whether he considers or he disregards the spatial aspect of the problem. This result is completely different from that obtained in the non-cooperative case. Second, in the more realistic case in which the regions value differently the environmental damage caused by the pollution stocks, the spatial aspect is essential in the social planner's decision making process. In this case, the strategic interactions among the players have disappeared, but the social planner can make decisions in a strategic manner from a spatial point of view.

\section{Concluding remarks}

This paper analyzes a transboundary pollution differential game where, in addition to the standard temporal dimension, a spatial dimension is introduced to capture the different geographical relationships among regions. There is a fairly recent literature devoted to the analysis of different economic and environmental problems by means of dynamic models that include the spatial dimension. However, most of this literature either neglects the strategic interactions among decision makers or neglects the dynamic aspect of the model. In the first case, the papers focus on the problem of a social planner; while in the second case, the decision-makers behave myopically in both the temporal and the spatial dimensions, and hence, agents solve static problems. As far as we know De Frutos \& Martín-Herrán (2017) is the only recent study that considers both the temporal and spatial dimensions of the problem as well as decision makers who behave both dynamically and strategically. In the present paper we follow the same approach and characterize the equilibrium outcomes of an intertemporal pollution problem where there is a continuum of spatial sites and the pollution stock diffuses over these sites. The functional specifications of the present work allow us to analytically treat the conditions that characterize the Markov-perfect Nash equilibrium of the space-discretized differential game. With the particular functional forms considered in this paper, borrowed from Jørgensen \& Zaccour (2001), the dynamic game belongs to the class of linear-state differential games. For this class of games it is well-known that the Markov-perfect Nash equilibria can be analytically characterized, although they are degenerated in the sense that they are constant over time. The simplest specification has been considered in order to be able to evaluate the effect of the spatial diffusion of the pollution stock in the definition of the environmental policies. In De Frutos \& Martín-Herrán (2017) the results are illustrated by means of numerical experiments even for the simplest case of two regions. Furthermore, our present functional specification allows the regions to invest in clean technology in order to reduce the emission-output ratio. Hence, the present specification allows us to analyze the effect of this new technology on the optimal emission strategies and the stock of pollution.

Our analytical results show how the equilibrium emission policies in a spatial context differ from those characterized ignoring the spatial dimension. In the non-cooperative framework, the spatially non-myopic behavior prescribes lower equilibrium emission rates, and as a consequence, a lower global pollution stock. However, this behavior is compatible with greater long-run welfares. In the cooperative framework, although the strategic interaction among the players does not exist, the only 
decision-maker still makes spatially strategic decisions. Furthermore, both in the cooperative and non-cooperative frameworks, the comparison of the equilibrium emission policies we have obtained in our spatial differential game version and those obtained for the same model when the spatial aspects are disregarded allows us to show that our spatial model can be viewed as a generalization of the non-spatial model. The equilibrium of the non-spatial formulation can be reproduced as a limit case of the spatial differential game. Specifically, the equilibrium environmental policy of the spatial model coincides with the equilibrium policy of the non-spatial model when the diffusion parameter, that describes how pollution diffuses among regions, tends to infinity. In this case, the stocks of pollution in the regions are instantaneously mixed, as is implicitly assumed in the standard hypothesis in a non-spatial setting.

One first further step in the analysis could be to evaluate the impact of the adoption of cleaner technology on the equilibrium emission rates and the long-run value of the pollution stock when the spatial dimension is taken into account and the clean technology and the pollution stock are not separable state variables. In this new formulation there would be a greater interrelation between the clean technology and pollution stocks. The characterization of the equilibrium strategies in this case would require the development of new numerical methods. Recently, Benchekroun \& Ray Chaudhuri $(2014,2015)$ have shown that the adoption of a cleaner technology may imply that the countries respond by increasing their emissions resulting in an increase of pollution that may be detrimental to welfare. The strategic behavior of the players may lead at first glance to counterintuitive results when the free-riding effect is exacerbated (Benchekroun \& Martín-Herrán (2016) study this effect in a transboundary pollution game with myopic and farsighted players).

In the present formulation pollution has a local dimension as a direct consequence of the production of the consumption good in a particular region. Another possible extension could be to add a second dimension for the pollution and consider that pollution produced in other regions may also harm welfare. In this case, the environmental damage function would depend on the pollution over the entire spatial domain. In a different framework Camacho \& Pérez-Barahona (2015) introduced the local and global dimension of pollution in their study of optimal land use and environmental degradation. This analysis is one of the subjects of our future research.

\section{References}

[1] Anita, S., Capasso, V., Kunze, H., \& La Torre, D. (2013). Optimal control and long-run dynamics for a spatial economic growth model with physical capital accumulation and pollution diffusion. Applied Mathematics Letters, 26, 908-912.

[2] Anita, S., Capasso, V., Kunze, H., \& La Torre, D. (2015). Dynamics and optimal control in a spatially structured economic growth model with pollution diffusion and environmental taxation. Applied Mathematics Letters, 42, 36-40.

[3] Augeraud-Véron, E., Choquet, C., \& Comte, E. (2017). Optimal control for a groundwater pollution ruled by a convection-diffusion-reaction problem. Journal of Optimization Theory and Applications, 173, 941-966.

[4] Başar, T., \& Olsder, G.J. (1999). Dynamic Noncooperative Game Theory. Philadelphia: SIAM. 
[5] Benchekroun, H., \& Martín-Herrán, G. (2016). The impact of foresight in a transboundary pollution game. European Journal of Operational Research, 251, 300-309.

[6] Benchekroun, H., \& Ray Chaudhuri, A. (2014). Transboundary pollution and clean technologies.Resource and Energy Economics, 36, 601-619.

[7] Benchekroun, H., \& Ray Chaudhuri, A. (2015). Cleaner technologies and the stability of international environmental agreements. Journal of Public Economic Theory, 17(6), 887-915.

[8] Brito, P. (2004). The dynamics of growth and distribution in a spatially heterogenous world. WP13/2004/DE/UECE, Technical University of Lisbon.

[9] Brock, W., \& Xepapadeas, A. (2008). General pattern formation in recursive dynamic systems models in economics. MPRA Paper No. 12305, University of Munich.

[10] Brock, W., \& Xepapadeas, A. (2008). Diffusion-induced instability and pattern formation in infinite horizon recursive optimal control. Journal of Economic Dynamics \& Control, 32, $2745-2787$.

[11] Brock, W., \& Xepapadeas, A. (2010). Pattern formation, spatial externalities and regulation in coupled economic-ecological systems. Journal of Environmental Economics and Management, 59, 149-164.

[12] Brock, W., Xepapadeas, A., \& Yannacopoulos, A.N. (2014). Optimal agglomerations in dynamic economics. Journal of Mathematical Economics, 53, 1-15.

[13] Brock, W., Xepapadeas, A., \& Yannacopoulos, A.N. (2014). Optimal control in space and time and the management of environmental resources. Annual Reviews of Resource Economics 6, $33-68$.

[14] Boucekkine, R., Camacho, C., \& Zou, B. (2009). Bridging the gap between growth theory and the New Economic Geography: the spatial Ramsey Mmodel. Macroeconomic Dynamics, 13, $20-45$.

[15] Boucekkine, R., Camacho, C., \& Fabbri, G. (2013). Spatial dynamics and convergence: the spatial Ak Model. Journal of Economic Theory, 148, 2719-2736.

[16] Boucekkine, R., Camacho, C., \& Fabbri, G. (2013). On the optimal control of some parabolic partial differential equations arising in economics. Serdica Mathematical Journal, 39, 331-354.

[17] Camacho, C., \& Pérez-Barahona, A. (2015). Land use dynamics and the environment. Journal of Economic Dynamics \& Control, 52, 96-118.

[18] Camacho, C., Zou, B., \& Briani, M. (2008). On the dynamics of capital accumulation across space. European Journal of Operational Research, 186, 451-465.

[19] De Frutos, J., \& Martín-Herrán, G. (2015). Does flexibility facilitate sustainability of cooperation over time? A case study from environmental economics. Journal of Optimization Theory and Applications, 165(2), 657-677. 
[20] De Frutos, J., \& Martín-Herrán, G. (2017). Spatial effects and strategic behavior in a multiregional transboundary pollution game. Accepted in Journal of Environmental Economics and Management. https://dx.doi.org/10.1016/j.jeem.2017.08.001.

[21] Desmet, K., \& Rossi-Hansberg, E. (2010). On spatial dynamics. Journal of Regional Science, $50,43-63$.

[22] Desmet, K., \& Rossi-Hansberg, E. (2015). On spatial economic impact of global warming. Journal of Urban Economics, 88, 16-37.

[23] Dockner, E.J., Jørgensen, S., Long, N.V., \& Sorger, G. (2000). Differential Games in Economics and Management Science. Cambridge: Cambridge University Press.

[24] Fabbri, G. (2016). Ecological barriers and convergence: A note on geometry in spatial growth models. Journal of Economic Theory, 162, 114-136.

[25] Graß, D., \& Uecker, H. (2017). Optimal management and spatial patterns in a distributed shalow lake model. Electronic Journal of Differential Equations, 1 (2017).

[26] Haurie, H, Krawczyk, J.B, \& Zaccour, G. (2012). Games and Dynamic Games. Singapore: World Scientific.

[27] Jørgensen, S., Martín-Herrán, G., \& Zaccour, G. (2010). Dynamic games in the economics and management of pollution. Environmental Modeling and Assessment, 15, 433-467.

[28] Jørgensen, S., \& Zaccour, G. (2001). Incentive equilibrium strategies and welfare allocation in a dynamic game of pollution control. Automatica, 37, 29-36.

[29] La Torre, D., Liuzzi, D., \& Marsiglio, S. (2015). Pollution diffusion and abatement activities across space and over time. Mathematical Social Sciences, 78, 48-63.

[30] Mäler, K-G., \& De Zeeuw, A. (1998). The acid rain differential game. Environmental and Resource Economics, 12, 167-184.

[31] Van der Ploeg, F., \& De Zeeuw, A.J. (1992). International aspects of pollution control. Environmental and Resource Economics, 2, 117-139.

[32] Stockie, J.M. (2011). The mathematics of atmospheric modeling. SIAM Review, 53, 349-372.

[33] Xepapadeas, A. (2010). The spatial dimension in environmental and resource economics. Environment and Development Economics, 15, 747-758. 


\section{Appendix A. Proofs of Propositions and Corollaries}

\section{Proof of Proposition 1}

The value function for player $i, V_{i}=V_{i}(\boldsymbol{p}, \boldsymbol{K}), i=1, \ldots, J$, satisfies the stationary HamiltonJacobi-Bellman equations

$$
\rho V_{i}=\max _{I_{i}, e_{i}}\left\{\log \left(e_{i}\right)+\beta_{i} K_{i}-\frac{1}{2} c_{i} I_{i}^{2}-\varphi_{i} p_{i}+\nabla_{\boldsymbol{K}} V_{i}(\boldsymbol{I}-\Gamma \boldsymbol{K})+\nabla_{\boldsymbol{p}} V_{i}(\mathrm{~B} \boldsymbol{p}-\mathrm{D}(\boldsymbol{p}-\boldsymbol{q})+\Pi \boldsymbol{e})\right\},
$$

where $\nabla_{\boldsymbol{K}} V_{i}$ and $\nabla_{\boldsymbol{p}} V_{i}$ denote the gradients of $V_{i}$ with respect to the variables $K_{1}, \ldots, K_{J}$ and $p_{1}, \ldots, p_{J}$, respectively.

Therefore, we postulate

$$
V_{i}(\boldsymbol{p}, \boldsymbol{K})=\boldsymbol{M}_{i}^{T} \boldsymbol{K}+\boldsymbol{R}_{\boldsymbol{i}}^{T} \boldsymbol{p}+X_{i},
$$

with $X_{i}$ constant and $\boldsymbol{M}_{i}=\left[M_{i, 1}, \ldots, M_{i, J}\right]^{T}$ and $\boldsymbol{R}_{\boldsymbol{i}}=\left[R_{i, 1}, \ldots, R_{i, J}\right]^{T}$ constant vectors to be determined.

The first-order conditions in (A.1) proportionate the values for the equilibrium investment and emissions rates in (15). Substituting in (A.1) and using the linearity of the value function we get that $M_{i, j}=0$ for $j \neq i$ and $M_{i, i}=\beta_{i} /\left(\rho+\mu_{i}\right)$. Coefficients $\boldsymbol{R}_{i}, i=1, \ldots, J$, satisfy the system of linear equations in (17).

System (17) possesses a unique solution because matrix $\mathrm{B}-\rho \mathrm{Id}-\mathrm{D}$ is strictly diagonally dominant. Furthermore, as the diagonal terms of $\mathrm{B}-\rho \mathrm{Id}-\mathrm{D}$ are strictly negative, matrix $\mathrm{B}-\rho \mathrm{Id}-\mathrm{D}$ is negative definite which proves that $R_{i, i}$ can be expressed as in (16) and, in consequence $e_{i}>0$, $i=1, \ldots, J$.

The constant term $X_{i}$ in (A.2) has the expression in (19).

The stationary equilibrium of the dynamics (13), (14) subject to (15) is globally asymptotically stable because the matrix system $B-D$ is negative definite if at least one of the $d_{i}, i=1, \ldots, J$ is different from zero.

\section{Proof of Proposition 2}

The sufficient condition for a stationary feedback Nash equilibrium requires us to find bounded and continuously differentiable functions, denoted by $V_{i}\left(K_{i}, p_{i}, p_{j}\right)$, which satisfies, for all $K_{i}(t)$, $p_{i}(t), p_{j}(t) \geq 0$, the Hamilton-Jacobi-Bellman (HJB) equations for both players. We first concentrate on finding solutions for the HJB equations. These equations are given by

$$
\begin{aligned}
\rho V_{i}\left(K_{i}, p_{i}, p_{j}\right) & =\max _{I_{i} \geq 0, e_{i} \geq 0}\left[\log \left(e_{i}\right)+\beta_{i} K_{i}-\frac{1}{2} c_{i} I_{i}^{2}-\varphi_{i} p_{i}+\frac{\partial V_{i}}{\partial K_{i}}\left(I_{i}-\mu_{i} K_{i}\right)\right. \\
& +\frac{\partial V_{i}}{\partial p_{i}}\left(b\left(p_{j}-p_{i}\right)-d_{i}\left(p_{i}-q_{i}\right)+\eta_{i} e_{i}\right) \\
& \left.+\frac{\partial V_{i}}{\partial p_{j}}\left(b\left(p_{i}-p_{j}\right)-d_{j}\left(p_{j}-q_{j}\right)+\eta_{j} e_{j}\right)\right] .
\end{aligned}
$$

The first-order optimality conditions read

$$
I_{i}\left(K_{i}, p_{i}, p_{j}\right)=\frac{\frac{\partial V_{i}}{\partial K_{i}}}{c_{i}}, \quad e_{i}\left(K_{i}, p_{i}, p_{j}\right)=-\frac{1}{\frac{\partial V_{i}}{\partial p_{i}} \eta_{i}}
$$


if these expressions are positive, and zero otherwise.

Guided by the model's linear-state structure, we assume that the players' value functions are linear functions in the state variables and given by (21). Taking into account this specification for players' value functions in (A.4) and inserting these optimal strategies into (A.3), the eight equations that characterize the coefficients of the value functions $M_{i, i}, R_{i, i}, R_{i, j}, X_{i}, i=1,2, i \neq j$ are determined by identification. These equations read as follows:

$$
\begin{aligned}
\left(\rho+\mu_{i}\right) M_{i, i}-\beta_{i} & =0, \\
\left(b+d_{i}+\rho\right) R_{i, i}-b R_{i, j}+\varphi_{i} & =0, \\
\left(b+d_{j}+\rho\right) R_{i, j}-b R_{i, i} & =0, \\
1-\log \left(-\frac{1}{R_{i, i} \eta_{i}}\right)+\rho X_{i}-\frac{M_{i, i}^{2}}{2 c_{i}}+\frac{R_{i, i}}{R_{j, i}}-R_{i, i} q_{i} d_{i}-R_{i, j} q_{j} d_{j} & =0 .
\end{aligned}
$$

From these equations the expressions of $M_{i, i}, R_{i, i}, R_{i, j}, X_{i}, i=1,2, i \neq j$ in the statement of the Proposition can be easily derived.

A sufficient condition guaranteeing that the expressions in (21) are the players' value functions and that (20) are the investment and emission strategies is given by

$$
\lim _{t \rightarrow \infty} e^{-\rho t} V_{i}\left(K_{i}(t), p_{i}(t), p_{j}(t)\right)=0
$$

where $\left(K_{i}(t), p_{i}(t), p_{j}(t)\right)$ is the solution of the closed-loop dynamics obtained after substituting the optimal investment and emission strategies (20) into the clean technology and pollution stocks dynamics given by (10) and

$$
\dot{p}_{i}=b\left(p_{j}-p_{i}\right)-d_{i}\left(p_{i}-q_{i}\right)+\eta_{i} e_{i}, \quad i, j=1,2, i \neq j .
$$

The steady-state levels of the clean technology and pollution stocks can be easily obtained replacing the optimal investment and emission strategies given by (20). The eigenvalues of the matrix associated with the closed-loop linear dynamical system are:

$$
-\mu_{i},-\mu_{j}, \frac{1}{2}\left(-\left(2 b+d_{i}+d_{j}\right) \pm \sqrt{4 b^{2}+\left(d_{i}+d_{j}\right)^{2}}\right) .
$$

The four eigenvalues are negative and hence, the steady-state equilibrium is globally asymptotically stable.

The linear functional specifications in (21) allow conditions in (A.5) to be satisfied whenever the state variables, the clean technology and pollution stocks, are bounded. This condition is guaranteed because the steady state is globally asymptotically stable.

\section{Proof of Corollary 1}

The results immediately follows from

1.

$$
\frac{\partial e_{i}}{\partial b}=\frac{\left(d_{j}+\rho\right)^{2}}{\eta_{i}\left(b+d_{j}+\rho\right)^{2} \varphi_{i}}>0, \quad i, j=1,2, i \neq j .
$$


2 .

$$
\frac{\partial p_{i}^{S S}}{\partial q_{i}}=\frac{d_{i}\left(b+d_{j}\right)}{d_{i} d_{j}+b\left(d_{i}+d_{j}\right)}>0, \quad \frac{\partial p_{i}^{S S}}{\partial q_{j}}=\frac{b d_{j}}{d_{i} d_{j}+b\left(d_{i}+d_{j}\right)}>0, \quad i, j=1,2, i \neq j .
$$

3.

$$
\left.\frac{\partial p_{i}^{S S}}{\partial b}\right|_{\substack{d_{i}=d_{j}=d \\ q_{i}=q_{j}=q \\ \varphi_{i}=\varphi_{j}=\varphi}}=\frac{(d+\rho)^{2}}{\eta_{i} d(b+d+\rho)^{2} \varphi}>0, \quad i=1,2 .
$$

\section{Proof of Corollary 2}

$$
\left.\frac{\partial p_{i}^{S S}}{\partial b}\right|_{\substack{d_{i}=d_{j}=d \\ q_{i}=q_{j}=q}}=\frac{(d+\rho) \Xi\left(b, d, \rho, \varphi_{i}, \varphi_{j}\right)}{d(2 b+d)^{2}(b+d+\rho)^{2} \varphi_{i} \varphi_{j}}, \quad i, j=1,2, i \neq j
$$

where

$$
\Xi\left(b, d, \rho, \varphi_{i}, \varphi_{j}\right)=4 b d(d+\rho) \varphi_{i}+d(d+\rho)\left(d \varphi_{i}+\rho\left(\varphi_{i}-\varphi_{j}\right)\right)+2 b^{2}\left(2 d \varphi_{i}+\rho\left(\varphi_{i}+\varphi_{j}\right)\right) .
$$

If $\varphi_{i}-\varphi_{j} \geq 0$, then $\Xi\left(b, d, \rho, \varphi_{i}, \varphi_{j}\right)>0$, and $\left.\frac{\partial p_{i}^{S S}}{\partial b}\right|_{\substack{d_{i}=d_{j}=d \\ q_{i}=q_{j}=q}}>0$.

If $\varphi_{i}-\varphi_{j}<0$ and $b$ is large, then $\Xi\left(b, d, \rho, \varphi_{i}, \varphi_{j}\right)>0$, and $\left.\frac{\partial p_{i}^{S S}}{\partial b}\right|_{\substack{d_{i}=d_{j}=d \\ q_{i}=q_{j}=q}}>0$.

If $\varphi_{i}-\varphi_{j}<0$ and $b$ is small, then the sign of $\Xi\left(b, d, \rho, \varphi_{i}, \varphi_{j}\right)$ coincides with the sign of $d \varphi_{i}+\rho\left(\varphi_{i}-\varphi_{j}\right)$. Hence, the last result immediately follows.

\section{Proof of Proposition 3}

The proof of this proposition follows the same steps as in the proof of Proposition 2.

\section{Proof of Proposition 4}

The results can be derived straightforwardly from Propositions 2 and 3 taking into account the expressions of $\widetilde{P}^{S S}$ and $\widetilde{e}_{i}$ as well as the following differences

$$
\begin{aligned}
e_{i}-\widetilde{e}_{i} & =-\frac{(d+\rho)^{2}}{2 \tilde{\eta}_{i}(b+d+\rho) \varphi_{i}}, \\
\widetilde{P}^{S S}-\frac{p_{i}^{S S}+p_{j}^{S S}}{2} & =\frac{(d+\rho)^{2}\left(\varphi_{i}+\varphi_{j}\right)}{2 d(b+d+\rho) \varphi_{i} \varphi_{j}},
\end{aligned}
$$

The gaps above tend to zero as the diffusion parameter $b$ goes to infinity.

\section{Proof of Proposition 5}

Under hypotheses $\mathbf{H}$ the following equalities apply:

$$
e_{i}=\frac{2 b+d+\rho}{2(b+d+\rho)} \widetilde{e}_{i}, \quad \frac{p_{i}^{S S}+p_{j}^{S S}}{2}=\frac{2 b+d+\rho}{2(b+d+\rho)} \widetilde{P}^{S S} .
$$

The ratio $\frac{2 b+d+\rho}{2(b+d+\rho)}$ monotonously increases with the diffusion parameter $b$, converges to one half as $b$ tends to zero and converges to one as $b$ goes to infinity. Therefore, $e_{i}$ and $\frac{p_{i}^{S S}+p_{j}^{S S}}{2}$ runs between the lower and upper bounds given in items i) and ii). Furthermore, the ratio decreases with the natural regeneration rate of pollution, $d$, and the temporal discount rate, $\rho$.

\section{Proof of Proposition 6}


The difference of the long-run discounted net welfares reads:

$$
\begin{aligned}
\widetilde{V}_{i}\left(K_{i}^{S S}, \widetilde{P}^{S S}\right)-V_{i}\left(K_{i}^{S S}, p_{i}^{S S}, p_{j}^{S S}\right) \\
\quad=\frac{1}{\rho} \log \left(\frac{2(b+d+\rho)}{2 b+d+\rho}\right)-\frac{(d+\rho)\left[d(d+\rho) \varphi_{i}+b\left(2 d+\rho\left(\varphi_{i}+\varphi_{j}\right)\right)\right]}{d \rho \varphi_{j}(2 b+d)(b+d+\rho)},
\end{aligned}
$$

and item i) immediately follows.

For the completely symmetric scenario $\left(\varphi_{i}=\varphi_{j}=\varphi\right)$, the difference above simplifies

$$
\widetilde{V}_{i}\left(K_{i}^{S S}, \widetilde{P}^{S S}\right)-V_{i}\left(K_{i}^{S S}, p_{i}^{S S}, p_{j}^{S S}\right)=\frac{1}{\rho} \log \left(\frac{2(b+d+\rho)}{2 b+d+\rho}\right)-\frac{(d+\rho)^{2}}{d \rho(b+d+\rho)} .
$$

It can be easily proved that the RHS of the equation above increases with $b$, takes a negative value for $b=0$ and tends to zero as $b$ converges to infinity, and as a result, the difference is always negative.

\section{Proof of Corollary 3}

The results in both items can be easily derived taking the corresponding limit in the expression of the difference $\widetilde{V}_{i}\left(K_{i}^{S S}, \widetilde{P}^{S S}\right)-V_{i}\left(K_{i}^{S S}, p_{i}^{S S}, p_{j}^{S S}\right)$ given in (A.8).

\section{Proof of Proposition 7}

Following the same steps as in the proof of Proposition 2 the equations that characterize the coefficients of the value function $M^{c}, N^{c}, R^{c}, T^{c}, X^{c}$ are determined by identification. These equations read as follows:

$$
\begin{aligned}
\left(\rho+\mu_{i}\right) M^{c}-\beta_{i} & =0, \\
\left(\rho+\mu_{j}\right) N^{c}-\beta_{j} & =0, \\
\left(b+d_{i}+\rho\right) R^{c}-b T^{c}+\varphi_{i} & =0, \\
\left(b+d_{j}+\rho\right) T^{c}-b R^{c}+\varphi_{j} & =0, \\
2-\log \left(-\frac{1}{R^{c} \eta_{i}}\right)-\log \left(-\frac{1}{T^{c} \eta_{j}}\right)+\rho X^{c}-\frac{\left(M^{c}\right)^{2}}{2 c_{i}}-\frac{\left(N^{c}\right)^{2}}{2 c_{j}}-R^{c} q_{i} d_{i}-T^{c} q_{j} d_{j} & =0 .
\end{aligned}
$$

From these equations the expressions of $M^{c}, N^{c}, R^{c}, T^{c}, X^{c}$ in the statement of the Proposition can be easily derived. Once these coefficients have been determined, the steady-state levels of clean technology and pollution stocks are immediately computed.

\section{Proof of Proposition 8}

The proof follows the same steps as in the proof of Proposition 7.

\section{Proof of Proposition 9}

From Propositions 7 and 8 we derive the following gaps:

$$
\begin{aligned}
e_{i}^{c}-\widetilde{e}_{i}^{c} & =-\frac{(d+\rho)^{2}\left(\varphi_{i}-\varphi_{j}\right)}{2 \tilde{\eta}_{i}\left(\varphi_{i}+\varphi_{j}\right)\left((b+d+\rho) \varphi_{i}+b \varphi_{j}\right)}, \\
\widetilde{P}^{S S c}-\frac{p_{i}^{S S c}+p_{j}^{S S c}}{2} & =-\frac{(d+\rho)^{3}\left(\varphi_{i}-\varphi_{j}\right)^{2}}{2 d\left(\varphi_{i}+\varphi_{j}\right)\left((b+d+\rho) \varphi_{i}+b \varphi_{j}\right)\left((b+d+\rho) \varphi_{j}+b \varphi_{i}\right)} .
\end{aligned}
$$


These gaps tend to zero as the diffusion parameter $b$ tends to infinity.

\section{Proof of Proposition 10}

Under hypotheses $\mathbf{H}$ the following equalities apply:

$$
\begin{aligned}
e_{i}^{c} & =\frac{(2 b+d+\rho)\left(\varphi_{i}+\varphi_{j}\right)}{2\left((d+\rho) \varphi_{i}+b\left(\varphi_{i}+\varphi_{j}\right)\right)} \widetilde{e}_{i}^{c}=\Lambda_{i} \widetilde{e}_{i}^{c} \\
\frac{p_{i}^{S S c}+p_{j}^{S S c}}{2} & =\frac{(2 b+d+\rho)^{2}\left(\varphi_{i}+\varphi_{j}\right)^{2}}{4\left((d+\rho) \varphi_{i}+b\left(\varphi_{i}+\varphi_{j}\right)\right)\left((d+\rho) \varphi_{j}+b\left(\varphi_{i}+\varphi_{j}\right)\right)} \widetilde{P}^{S S c}=\Lambda^{c} \widetilde{P}^{S S c} .
\end{aligned}
$$

The ratio $\Lambda_{i}$ monotonously increases with the diffusion parameter $b$ if $\varphi_{i}>\varphi_{j}$ and decreases with $b$ if $\varphi_{i}<\varphi_{j}$. The ratio $\Lambda_{i}$ converges to $\frac{\varphi_{i}+\varphi_{j}}{2 \varphi_{i}}$ as $b$ tends to zero and converges to one as $b$ goes to infinity. Furthermore, the ratio $\Lambda_{i}$ decreases (increases) with $d$ and $\rho$ if $\varphi_{i}$ is greater (lower) than $\varphi_{j}$.

The ratio $\Lambda^{c}$ decreases with the diffusion parameter $b$ and increases with $d$ and $\rho$. The ratio $\Lambda^{c}$ tends to $\frac{\left(\varphi_{i}+\varphi_{j}\right)^{2}}{4 \varphi_{i} \varphi_{j}}$ as $b$ tends to zero and converges to 1 as $b$ tends to infinity. Therefore, $e_{i}^{c}$ and

$\frac{p_{i}^{c}+p_{j}^{S S c}}{2}$ runs between the lower and upper bounds given in items i) and ii). Furthermore, the ratio decreases with $d$ and $\rho$.

\section{Proof of Proposition 11}

The difference of the long-run cooperative discounted net welfares reads:

$$
\begin{aligned}
& V^{c}\left(K_{i}^{S S c}, K_{j}^{S S c}, p_{i}^{S S c}, p_{j}^{S S c}\right)-\widetilde{V}^{c}\left(K_{i}^{S S c}, K_{j}^{S S c}, \widetilde{P}^{S S c}\right)= \\
& \quad \frac{(2 b+d+\rho)^{2}\left(\varphi_{i}+\varphi_{j}\right)^{2}}{\rho} \log \left(\frac{(d+\rho)^{2}\left(\varphi_{i}-\varphi_{j}\right)^{2}}{\left.4\left((d+\rho) \varphi_{i}+b\left(\varphi_{i}+\varphi_{j}\right)\right)\left((d+\rho) \varphi_{j}+\varphi_{j}\right)\right)}\right) \\
& \quad-b \frac{\left.(d+\rho) \varphi_{j}+b\left(\varphi_{i}+\varphi_{j}\right)\right)}{d(2 b+d)\left((d+\rho) \varphi_{i}+b\left(\varphi_{i}+\varphi_{j}\right)\right)((d+\rho)}
\end{aligned}
$$

From the expression above the proof easily follows.

\section{Proof of Corollary 4}

It is easy to show that the difference $V^{c}\left(K_{i}^{S S c}, K_{j}^{S S c}, p_{i}^{S S c}, p_{j}^{S S c}\right)-\widetilde{V}^{c}\left(K_{i}^{S S c}, K_{j}^{S S c}, \widetilde{P}^{S S c}\right)$ given in (A.11) is positive as $b$ tends to zero. Easy but tedious computations show that the sign of the derivative of this difference with respect to $b$ coincides with the sign of a fourth-order polynomial in $b$. If $d \geq 2 \rho$ all the coefficients of the polynomial are negative, and hence, the difference monotonously decreases as $b$ increases from a positive value to zero, which proves the first item. If $d<2 \rho$ the coefficients of the polynomial present different signs. The second term follows because the independent term is negative and the coefficient of the highest order is positive. 